\title{
A numerical evaluation of the asymptotic theory of receptivity for subsonic compressible boundary layers
}

\author{
NICOLA DE TULLIO, ANATOLY I. RUBAN \\ Department of Mathematics, Imperial College London, South Kensington Campus, \\ London, SW7 2AZ, UK
}

(Received ?? and in revised form ??)

The capabilities of the triple-deck theory of receptivity for subsonic compressible boundary layers have been thoroughly investigated through comparisons with numerical simulations of the compressible Navier-Stokes equations. The analysis focused on the two Tollmien-Schlichting wave linear receptivity problems arising due to the interaction between a low amplitude acoustic wave and a small isolated roughness element and the low amplitude, time-periodic vibrations of a ribbon placed on the wall of a flat plate. A parametric study was carried out to look at the effects of roughness element and vibrating ribbon longitudinal dimensions, Reynolds number, Mach number and TollmienSchlichting wave frequency. The flat plate is considered isothermal, with a temperature equal to the laminar adiabatic-wall temperature. Numerical simulations of the full and the linearised compressible Navier-Stokes equations have been carried out using highorder finite differences to obtain, respectively, the steady basic flows and the unsteady disturbance fields for the different flow configurations analysed. The results show that the asymptotic theory and the Navier-Stokes simulations are in good agreement. The initial Tollmien-Schlichting wave amplitudes and, in particular, the trends indicated by the theory across the whole parameter space are in excellent agreement with the numerical results. An important finding of the present study is that the behaviour of the theoretical solutions obtained for $R e \rightarrow \infty$ holds at finite Reynolds numbers and the only conditions needed for the theoretical predictions to be accurate are that the receptivity process be linear and the free stream Mach number be subsonic.

Key Words:

\section{Introduction}

In "quiet" disturbance environments, which are typical of flight conditions, the initial stages of laminar-turbulent transition are dominated by the evolution of instability modes (primary instabilities, possibly followed by secondary instabilities), which grow exponentially due to linear processes. The nature of these instabilities depends greatly on the state of the laminar boundary layer. In boundary layers developing over swept wings with large sweep angles the transition process is dominated by the Cross-Flow instability. The Tollmien-Schlichting (T-S) waves drive the boundary layer instability in the case of small sweep angles, while Taylor-Görtler vortices are the dominant primary instability for boundary layers developing over concave walls. Since the first pioneering experiments of Reynolds (1883), the complexity and great variety of possible mechanisms involved in the laminar-turbulent transition has inspired a great deal of work on this subject, despite 
which transition prediction still remains a formidable task. The main problem associated with current transition prediction methodologies is that they are largely based on the concept of disturbance amplification, rather than on the disturbance amplitude, and hence completely disregard the receptivity process, the importance of which was highlighted by numerous experiments (see Reshotko 1976; Kachanov 1994; Saric et al. 2002, and references therein). Receptivity theory studies the process of interaction between the boundary layer and "external perturbations", such as acoustic waves, free-stream turbulence and body surface vibrations. The objective of the receptivity analysis is, first, to identify how the external perturbations can be converted into instability modes of the boundary layer, the T-S waves, Cross-Flow vortices or Taylor-Görtler vortices, and, second, to determine the initial amplitude of these modes.

The progress achieved in this field has been, to a large degree, thanks to the studies based on the asymptotic analysis of the Navier-Stokes equations at large values of the Reynolds number. When dealing with the process of generation of T-S waves in boundary layers, this approach relies on the triple-deck theory, which is known to describe the T-S waves in subsonic flows near the lower branch of the neutral stability curve (see Lin 1946; Smith $1979 a, b)$. The first paper where the triple-deck theory was used to study the receptivity of the boundary layer was published by Terent'ev (1981). He considered an incompressible flow past a flat plate with the basic steady flow given by the Blasius solution. He assumed that a short section of the plate surface performs periodic vibrations in the direction perpendicular to the wall. This formulation represents a simplified mathematical model of the classical experiments performed by Schubauer \& Skramstad (1948) where the T-S waves were generated by a vibrating ribbon installed a small distance above the plate surface. Terent'ev's theory shows that, in the vicinity of the vibrating part of the wall, the perturbation field is rather complex. However, further downstream only one perturbation mode survives, the T-S wave. The amplitude of this wave depends on the shape of the vibrating part of the wall.

It is known from numerous observations that the boundary layers are susceptible to acoustic noise. Asymptotic theory of the generation of T-S waves by acoustic noise, was developed by Ruban (1984) and Goldstein (1985). In these studies the importance of notion of "double resonance" was highlighted as a fundamental principle of the receptivity theory. In fluid flows, effective transformation of external disturbances into instability modes of the boundary layer is only possible if, in addition to the frequency, the wave number of the external perturbations is in tune with the natural internal oscillations of the boundary layer. These conditions could be easily satisfied in the problem considered by Terent'ev (1981), where the frequency and the length of the vibrating part of the wall can be chosen independent of one another. When an acoustic wave impinges upon the boundary layer, the pressure perturbations in the acoustic wave penetrate into the boundary layer leading to the creation of a near-wall Stokes layer inside the boundary layer. If the acoustic field has a wide enough spectrum, then the receptivity process will "extract" from it a harmonic whose frequency is in tune with the frequency of the corresponding T-S wave. Of course, under this condition the wavelength of the "chosen" acoustic wave appears to be much longer than that of the T-S wave, meaning that the second resonance condition, the tuning of the wavenumbers, is not satisfied. However, in practical applications, such as in the flow past an aircraft wing, the body surface in never absolutely smooth. Hence, in addition to unsteady perturbations in the Stokes layer, one has to consider steady perturbations produced by the wall roughnesses, which normally have a short length scale. Ruban and Goldstein demonstrated that the interaction between the two perturbation modes leads to the formation of T-S waves behind the roughness. Along with acoustic waves, free-stream turbulence is also known to have a significant influence 
on the laminar-turbulent transition in the boundary layer. The asymptotic theory of the receptivity of the boundary layer to the free-stream turbulence was developed by Duck et al. (1996). They found that, unlike the acoustic waves, the vorticity waves do not carry pressure perturbations, and therefore are unable to penetrate into the boundary layer. However, the steady flow perturbations produced by a wall roughness are not confined to the boundary layer but extend to the flow outside the boundary layer, where they come into interaction with the vorticity wave. Duck et al. (1996) showed that this interaction results in a T-S wave forming downstream of the roughness.

Recently, the generation of T-S waves in the boundary layer due to elastic vibrations of the wing surface was analysed by Ruban et al. (2013). Their results show that the wing surface vibrations can cause pressure perturbations in the flow outside the boundary layer, which, in turn, induce a Stokes layer near the wing surface. Two physical mechanisms were found to be able to induce an oscillatory motion of the Stokes layer. The first one is the classical mechanism where the pressure gradient, being a periodic function of time, forces the fluid to oscillate in the direction along the wing surface. This process is similar to the one describe by Ruban (1984) and Goldstein (1985) in their study of the boundary layer receptivity to acoustic waves. In the second mechanism the pressure itself, not the pressure gradient, makes up the Stokes layer. In both cases, T-S waves are generated when the Stokes layer encounters a wall roughness. These and other examples (Denier et al. 1991; Wu 2001; Kerimbekov \& Ruban 2005; Wu et al. 2011) show that the asymptotic approach has proven to be invaluable in uncovering possible mechanism of the boundary-layer receptivity. However, the accuracy, with which the asymptotic theory predicts the initial amplitude of the instability modes forming in the boundary layer, has been under question.

Boundary layer receptivity problems have also been extensively analysed experimentally (see for example Kachanov et al. 1979; Saric \& White 1998; Dietz 1999; Borodulin et al. 2013) and numerically (see for example Fucciarelli et al. 2000; Wanderley \& Corke 2001; Jones et al. 2010; Tempelmann et al. 2012). The main challenge of the experimental investigations is the measurement of the receptivity coefficients, since the initial amplitudes of the boundary layer instabilities may be orders of magnitude smaller than the amplitude of the surrounding disturbance environment. On the other hand, the main difficulty associated with numerical simulations is to accurately represent the free stream disturbance environment, whereby care needs to be taken to correctly formulate the boundary conditions needed to capture the effects of the different types of perturbations (acoustic waves, entropy waves and vorticity waves) on the boundary layer receptivity. Despite the numerous efforts, comparisons between the predictions of the asymptotic theory of receptivity and the numerical and experimental results are very limited. Comparisons with experiments can be found for example in Goldstein \& Hultgren (1987), Kozlov \& Ryzhov (1990) and Wu (2001), while detailed comparisons with the predictions of the finite-Reynolds number Orr-Sommerfeld theory (Zhigulev \& Fedorov 1987; Choudhari \& Street 1992; Crouch 1992) are reported in Choudhari \& Street (1992). However, the range of parameters over which the asymptotic theory has been compared with high-fidelity Navier-Stokes numerical simulations and/or experiments is very limited, hence a detailed evaluation of the capabilities of the theory is currently missing. In this paper we address this issue by comparing the asymptotic theory with numerical simulations of the compressible Navier-Stokes equations, focusing on two important receptivity problems: the T-S wave generation by a vibrating ribbon placed on the wall of a flat plate and that due to the interaction between a small isolated roughness element and an acoustic wave travelling in the flow direction. The investigation is carried out through a parametric study on the effects of roughness element/vibrator longitudinal di- 
mension, Reynolds number, Mach number and T-S wave frequency. In this work, special care has been taken in the formulation of the boundary conditions used to obtain the relevant disturbance fields (particularly for the numerical analysis of the receptivity due to sound), which are critical for the accurate determination of the receptivity coefficients. The numerical techniques used to obtain the disturbance fields are described in $\S 4.2$.

The paper is organised as follows. In $\S 2$ the receptivity problems are introduced along with the theoretical preliminaries of the triple-deck study. The main steps of the tripledeck analysis are given in $\S 3$. All the details of the numerical study are given in $\S 4$. In $\S 5$ we provide comparisons between the numerical and the theoretical results, along with a discussion of the main findings. The paper ends in $\S 6$, where the main conclusions of the study are drawn.

\section{Problem formulation and triple-deck scalings}

Consider the two-dimensional laminar boundary layer that forms on the surface of a flat plate in a subsonic free stream. We shall analyse the two receptivity problems arising from the interaction between an acoustic wave and an isolated two-dimensional roughness element and from the time-periodic vibrations of a ribbon placed on the flat plate wall, as schematically depicted in figure 1 (note that, for brevity, in the remainder of this paper the roughness element and the vibrating ribbon will be referred to as "the wall disturbance" whenever the subject of the discussion applies to both). The mechanisms responsible for the generation of T-S waves in the above two scenarios are fully described by the compressible Navier-Stokes equations, which, for a two-dimensional flow, may be written as

$$
\begin{aligned}
& \frac{\partial \rho}{\partial t}+\frac{\partial \rho u}{\partial x}+\frac{\partial \rho v}{\partial y}=0, \\
& \rho \frac{D u}{D t}=-\frac{\partial p}{\partial x}+\frac{1}{R e} \frac{\partial}{\partial y}\left(\mu \frac{\partial u}{\partial y}\right)+\cdots, \\
& \rho \frac{D v}{D t}=-\frac{\partial p}{\partial y}+\frac{1}{R e} \frac{\partial}{\partial y}\left(\mu \frac{\partial v}{\partial y}\right)+\cdots, \\
& \rho \frac{D h}{D t}=\frac{D p}{D t}+\frac{1}{R e P r} \frac{\partial}{\partial y}\left(\mu \frac{\partial h}{\partial y}\right)+\frac{\mu}{R e}\left(\frac{\partial u}{\partial y}\right)^{2}+\cdots, \\
& p=\frac{\gamma-1}{\gamma} \rho h=\frac{\rho T}{\gamma M^{2}} .
\end{aligned}
$$

Note that, for brevity, only the principal viscous terms are shown in the momentum and energy equations. The equations are written in terms of the following non-dimensional variables

$$
\left.\begin{array}{l}
x=\frac{x^{*}-x_{0}^{*}}{L^{*}}, \quad y=\frac{y^{*}}{L^{*}}, \quad u=\frac{u^{*}}{U_{\infty}^{*}}, \quad v=\frac{v^{*}}{U_{\infty}^{*}}, \quad \rho=\frac{\rho^{*}}{\rho_{\infty}^{*}}, \\
p=\frac{p^{*}}{\rho_{\infty}^{*} U_{\infty}^{* 2}}, \quad \mu=\frac{\mu^{*}}{\mu_{\infty}^{*}}, \quad t=\frac{t^{*} U_{\infty}^{*}}{L^{*}}, \quad h=\frac{h^{*}}{U_{\infty}^{* 2}}, \quad
\end{array}\right\}
$$

where the reference length $L^{*}$ is the distance between the leading edge of the flat plate and the centre of the wall disturbance, which is positioned at $x^{*}=x_{0}^{*}$. The $x^{*}$ coordinate measures the distance along the flat plate wall starting from its leading edge and the $y^{*}$ coordinate indicates the distance to the wall of the plate. The streamwise and wall-normal velocities are denoted respectively as $u^{*}$ and $v^{*}$, the fluid density as $\rho^{*}$, the pressure as 


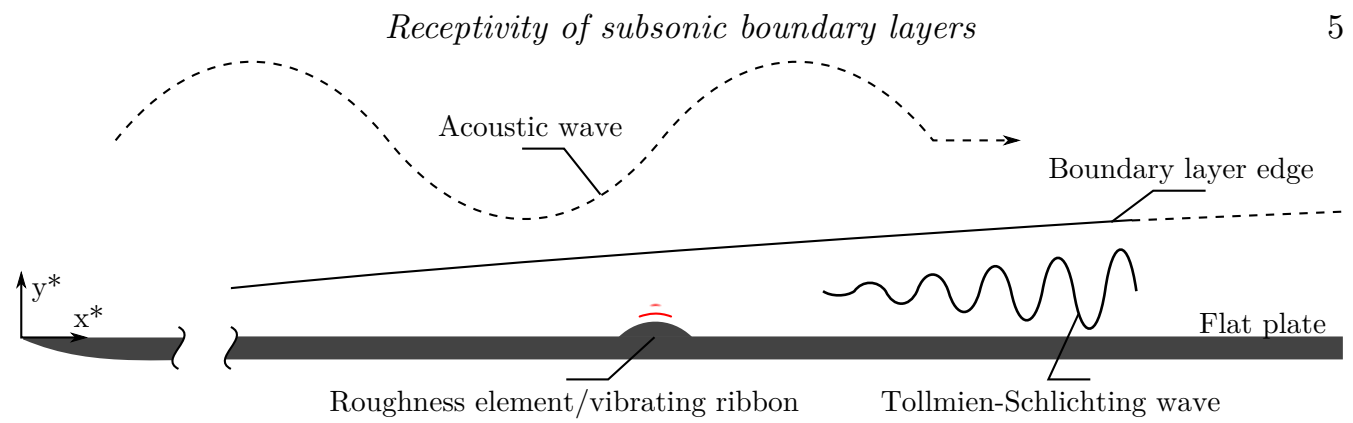

FiguRE 1. Schematic reppresentation of the receptivity problem.

$p^{*}$, the dynamic viscosity as $\mu^{*}$ and the enthalpy as $h^{*}$. Asterisks indicate dimensional quantities. The dimensionless parameters for this problem are the Reynolds number Re, the Mach number $M$ and the Prandtl number $P r$, which we consider to be constant and equal to $\operatorname{Pr}=0.72$.

In the absence of any external perturbations the laminar basic flow over a smooth flat plate is governed by the classical compressible boundary layer equations which, using Illingworth's transformation (see for example White 2005), may be written as

$$
\begin{aligned}
& \left(C \mathcal{F}^{\prime \prime}\right)^{\prime}+\mathcal{F} \mathcal{F}^{\prime \prime}=0, \\
& \left(C \mathcal{G}^{\prime}\right)^{\prime}+\operatorname{Pr} \mathcal{F} \mathcal{G}^{\prime}=-(\gamma-1) C \operatorname{Pr} M^{2} \mathcal{F}^{\prime \prime 2},
\end{aligned}
$$

where $\mathcal{F}^{\prime}(\eta)=U_{B}$ is the basic flow streamwise velocity, $\mathcal{G}(\eta)=h_{B}$ is the basic flow enthalpy and $C(\mathcal{G})=\mu_{B} \rho_{B}$ is the Chapman-Rubesin parameter. Equations (2.3) are obtained after introducing the similarity coordinates

$$
\xi=\mu_{\infty}^{*} \rho_{\infty}^{*} U_{\infty}^{*} L^{*}(1+x) \text { and } \eta=\frac{(1+x)^{-1 / 2}}{\sqrt{2}} \int_{o}^{Y} \rho \mathrm{d} Y,
$$

where $Y=R e^{1 / 2} y$ is the usual boundary layer wall normal coordinate. In this work we assume that the flat plate wall is adiabatic, so that the wall-temperature is constant and equal to

$$
T_{w}=1+\operatorname{Pr}^{1 / 2} \frac{\gamma-1}{2} M^{2} .
$$

Under this condition, equations (2.3) admit smooth self-similar solutions which may be expanded in Taylor series around $x=0$ (i.e. around the centre of the wall disturbance) as

$$
\left.\begin{array}{l}
U_{B}(x, Y)=U_{B 0}(Y)+\mathcal{O}(x), \\
\rho_{B}(x, Y)=\rho_{B 0}(Y)+\mathcal{O}(x), \\
h_{B}(x, Y)=h_{B 0}(Y)+\mathcal{O}(x), \\
\mu_{B}(x, Y)=\mu_{B 0}(Y)+\mathcal{O}(x)
\end{array}\right\} \text { as } x \rightarrow 0 \text { and } Y=\mathcal{O}(1)
$$

In addition, the near wall behaviour of the boundary layer flow near $x=0$ can also be recovered by noting that the leading order terms in (2.6) may in turn be Taylor expanded near the wall, hence one can write

$$
\left.\begin{array}{l}
U_{B}=\lambda Y+\cdots, \\
\rho_{B}=\rho_{w}+\cdots, \\
h_{B}=h_{w}+\cdots \\
\mu_{B}=\mu_{w}+\cdots
\end{array}\right\} \text { as } x \rightarrow 0 \text { and } Y \rightarrow 0
$$




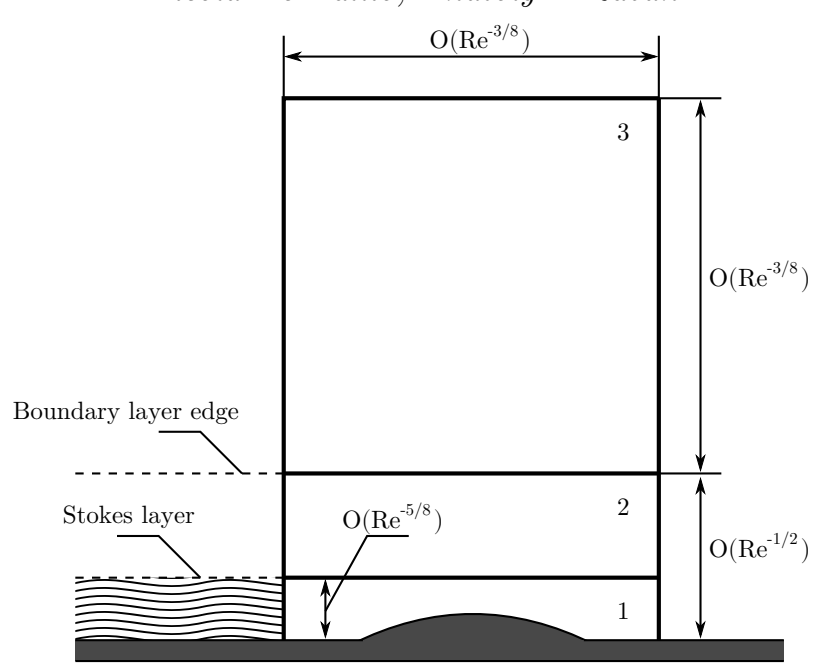

FiguRE 2. Triple-deck formalism.

where

$$
\lambda=\left.\frac{\mathrm{d} U_{B 0}}{\mathrm{~d} Y}\right|_{w}=\frac{\mathcal{F}^{\prime \prime}(0)}{\sqrt{2} T_{w}}
$$

and $\mathcal{F}^{\prime \prime}(0)$ is obtained by solving (2.3) numerically. The values of $\mathcal{F}^{\prime \prime}(0)$ associated with the numerical simulations carried out in this work are given in $\S 4.2$ (table 1).

As anticipated in the introduction, the asymptotic theory of receptivity is based on the triple-deck theory, which describes the T-S waves in subsonic flows near the lower branch of the neutral stability curve (see Lin 1946; Smith 1979a,b). Therefore, following the triple-deck formalism, the flow near the wall disturbance is divided into three regions in the wall normal direction, as depicted in figure 2. Strictly speaking, the wall disturbance should be contained within the lower-deck (region 1) in order for the triple-deck theory to be valid. In addition, the frequency of the vibrating ribbon and the free stream acoustic wave has to match the lower branch T-S wave frequency, which is an $\mathcal{O}\left(R e^{1 / 4}\right)$ quantity (Lin 1946). Therefore, the frequency of the imposed oscillations, the streamwise length of the wall disturbance and the wall-normal size $(h)$ of the wall disturbance (taken here as the amplitude of the vibrations of the ribbon or the height of the roughness element) are taken to be of $\mathcal{O}\left(R e^{1 / 4}\right), \mathcal{O}\left(R e^{-3 / 8}\right)$ and $\mathcal{O}\left(R e^{-5 / 8}\right)$, respectively.

Based on the above considerations the shape of the roughness element and the vibrating ribbon may be expressed respectively as

$$
y_{r}=h F\left(\frac{x}{R e^{-3 / 8}}\right) \quad \text { and } \quad y_{r}=h F\left(\frac{x}{R e^{-3 / 8}}\right) G\left(\frac{t}{R e^{-1 / 4}}\right),
$$

where $h=\epsilon R e^{-5 / 8}$ with $\epsilon=\mathcal{O}(1)$. In order to obtain an analytical description of the receptivity of T-S waves by the interaction between an acoustic wave and a roughness element, the disturbances induced inside the boundary layer by the acoustic wave also need to be analysed using asymptotic analysis. It can be shown that the acoustic wave leads to the generation of a thin oscillating layer near the wall, the Stokes layer, the thickness of which is of the same order of magnitude as that of the lower-deck, as schematically depicted in figure 2 .

The asymptotic theory of the generation of T-S waves by sound is due to Ruban (1984) and Goldstein (1985), while the receptivity problem of a vibrating ribbon was first solved 
in the context of the triple-deck theory by Terent'ev (1981). The aim of this paper is to provide a detailed assessment of the applicability of the above theories by comparing their predictions with high-fidelity Navier-Stokes simulation results. Therefore, only the relevant portions of the theoretical analyses are provided in the following for completeness.

\section{Triple-deck theory}

The flow in the vicinity of a wall disturbance given by one of equations (2.9) may be described by the triple-deck theory. In the lower deck, after introducing the usual scaled coordinates

$$
\bar{t}=R e^{1 / 4} \frac{\mu_{w}^{1 / 2}}{\lambda^{-3 / 2} \beta^{-1 / 2}} t, \quad \bar{x}=R e^{3 / 8} \frac{\mu_{w}^{1 / 4} \rho_{w}^{1 / 2}}{\lambda^{-5 / 4} \beta^{-3 / 4}} x \text { and } \bar{y}=R e^{5 / 8} \frac{\mu_{w}^{-1 / 4} \rho_{w}^{1 / 2}}{\lambda^{-3 / 4} \beta^{-1 / 4}} y
$$

and substituting the following asymptotic expansions

$$
\left.\begin{array}{l}
u=R e^{-1 / 8} \frac{\mu_{w}^{1 / 4} \rho_{w}^{-1 / 2}}{\lambda^{-1 / 4} \beta^{1 / 4}} \bar{U}+\cdots, \quad v=R e^{-3 / 8} \frac{\mu_{w}^{3 / 4} \rho_{w}^{-1 / 2}}{\lambda^{-3 / 4} \beta^{-1 / 4}} \bar{V}+\cdots, \\
p=p_{\infty}+R e^{-1 / 4} \frac{\mu_{w}^{1 / 2}}{\lambda^{-1 / 2} \beta^{1 / 2}} \bar{P}+\cdots,
\end{array}\right\}
$$

into the Navier-Stokes equations (2.1), the governing equations can be written as

$$
\begin{aligned}
& \frac{\partial \bar{U}}{\partial \bar{x}}+\frac{\partial \bar{V}}{\partial \bar{y}}=0 \\
& \frac{\partial \bar{U}}{\partial \bar{t}}+\bar{U} \frac{\partial \bar{U}}{\partial \bar{x}}+\bar{V} \frac{\partial \bar{U}}{\partial \bar{y}}=-\frac{\partial \bar{P}}{\partial \bar{x}}+\frac{\partial^{2} \bar{U}}{\partial \bar{y}^{2}}
\end{aligned}
$$

with the boundary conditions given by

$$
\begin{aligned}
& \bar{U}=\bar{U}_{w}, \bar{V}=\bar{V}_{w} \text { at } \bar{y}=\bar{y}_{r}, \\
& \bar{U}=\bar{y}+\cdots \text { as } \bar{x} \rightarrow-\infty, \\
& \bar{U}=\bar{y}+\bar{A}(\bar{t}, \bar{x})+\cdots \text { as } \bar{y} \rightarrow \infty .
\end{aligned}
$$

Here $\beta=\sqrt{1-M^{2}}, \bar{A}$ is the usual unknown displacement function and $\bar{U}_{w}$ and $\bar{V}_{w}$ represent the motion of the flat plate wall $\left(\bar{U}_{w}=\bar{V}_{w}=0\right.$ in the case of a steady roughness element). It is important to note that, since the local Mach number is small near the wall, we have assumed that the flow is incompressible and hence have neglected density, viscosity and enthalpy disturbances. Given the scaling introduced for the time $t$ and and the $x$-coordinate it is convenient to explicitly introduce the scaled frequency $\bar{\omega}=R e^{-1 / 4} \mu_{w}^{-1 / 2} \lambda^{-3 / 2} \beta^{-1 / 2} \omega$ and the scaled streamwise wavenumber $\bar{\alpha}=$ $R e^{-3 / 8} \mu_{w}^{-1 / 4} \rho_{w}^{-1 / 2} \lambda^{-5 / 4} \beta^{-3 / 4} \alpha$ for future reference.

\subsection{Receptivity of $T-S$ waves by sound}

In the lower-deck coordinates (3.1) the shape of the roughness element may be written as $\bar{y}_{r}=\epsilon \bar{F}(\bar{x})$, where we have used $F=\mu_{w}^{1 / 4} \rho_{w}^{-1 / 2} \lambda^{-3 / 4} \beta^{-1 / 4} \bar{F}$ and, since here we are interested in the linear case, we with put $\epsilon \ll 1$. Let us now assume that there is a plane acoustic wave travelling in the free stream in the flow direction. The acoustic wave amplitude is chosen so that the streamwise pressure gradient induced by the wave is of the same order of magnitude as that induced by the roughness element. It turns out that this condition is fulfilled if the acoustic wave amplitude is an order $\mathcal{O}\left(R e^{-1 / 8}\right)$ quantity; 
here we put $a=\delta R e^{-1 / 8}$, with $\delta \ll 1$. Such a wave induces a Stokes layer near the wall; the interaction between the Stokes layer disturbances and the roughness element gives rise to a T-S wave.

The disturbances introduced into the boundary layer in the vicinity of the roughness element are due to: the roughness element itself, denoted as say $u_{1}$; the oscillations of the Stokes layer, say $u_{s}$, and the interactions between the previous two, say $u_{2}$. Therefore, after applying Prandtl's transformations

$$
\left.\begin{array}{lll}
\bar{t}=\tilde{t}, & \bar{x}=\tilde{x}, & \bar{y}=\tilde{y}+\epsilon \tilde{F}, \\
\bar{U}=\tilde{U}, & \bar{V}=\tilde{V}+\epsilon \tilde{U} \tilde{F}^{\prime}, & \bar{P}=\tilde{P}, \\
\bar{F}=\tilde{F}, &
\end{array}\right\}
$$

which are used to simplify the wall boundary conditions, we look for lower-deck solutions in the following form

$$
\begin{aligned}
\tilde{U} & =\tilde{y}+\delta u_{s}\left(\tilde{t}, x_{s}, \tilde{y}\right)+\epsilon u_{1}(\tilde{x}, \tilde{y})+\epsilon \delta u_{2}(\tilde{t}, \tilde{x}, \tilde{y})+\cdots, \\
\tilde{V} & =R e^{-1 / 8} \delta v_{s}\left(\tilde{t}, x_{s}, \tilde{y}\right)+\epsilon v_{1}(\tilde{x}, \tilde{y})+\epsilon \delta v_{2}(\tilde{t}, \tilde{x}, \tilde{y})+\cdots, \\
\tilde{P} & =R e^{1 / 8} \delta p_{s}\left(\tilde{t}, x_{s}\right)+\epsilon p_{1}(\tilde{x})+\epsilon \delta p_{2}(\tilde{t}, \tilde{x})+\cdots,
\end{aligned}
$$

where $x_{s}=R e^{1 / 4} x$ and the Stokes layer terms (subscript $s$ ) are introduced to ensure that the solution matches the Stokes layer solution for $\tilde{x} \rightarrow-\infty$. By substituting the above into equations (3.3)-(3.4) we obtain a steady problem describing the roughness-induced disturbances $u_{1}$ (by collecting terms with $\epsilon$ ) and an unsteady problem describing the disturbances arising from the interaction between the Stokes layer and the roughness element (by collecting terms with $\epsilon \delta$ ).

In Fourier space, $\hat{u}_{1}=(2 \pi)^{-1 / 2} \int_{-\infty}^{\infty} u_{1} \mathrm{e}^{-\mathrm{i} \bar{\alpha} \tilde{x}} \mathrm{~d} \tilde{x}$, the steady problem has the solution $\hat{u}_{1}=\Gamma(\zeta ; \bar{\alpha}) \hat{F}(\bar{\alpha})$ and $\hat{v}_{1}=\Theta(\zeta ; \bar{\alpha}) \hat{F}(\bar{\alpha})$, where $\zeta=(\mathrm{i} \bar{\alpha})^{1 / 3} \tilde{y}$ and

$$
\begin{aligned}
& \Gamma(\zeta ; \bar{\alpha})=-\frac{3(\mathrm{i} \bar{\alpha})^{1 / 3}|\bar{\alpha}|}{3 \mathrm{Ai}^{\prime}(0)-|\bar{\alpha}|(\mathrm{i} \bar{\alpha})^{1 / 3}} \int_{0}^{\zeta} \mathrm{Ai}(s) \mathrm{d} s \\
& \Theta(\zeta ; \bar{\alpha})=-(\mathrm{i} \bar{\alpha})^{2 / 3} \int_{0}^{\zeta} \Gamma(s ; \bar{\alpha}) \mathrm{d} s .
\end{aligned}
$$

The unsteady problem admits time-harmonic solutions $u_{2}=1 / 2 \check{u}_{2} \exp (\mathrm{i} \tilde{\omega} \tilde{t})+$ c.c. and, by taking the Fourier transform $\hat{u}_{2}=(2 \pi)^{-1 / 2} \int_{-\infty}^{\infty} \check{u}_{2} \mathrm{e}^{-\mathrm{i} \bar{\alpha} \tilde{x}} \mathrm{~d} \tilde{x}$, can be expressed as

$$
\begin{aligned}
& \frac{\mathrm{d}^{3} \hat{u}_{2}}{\mathrm{~d} z^{3}}-z \frac{\mathrm{d} \hat{u}_{2}}{\mathrm{~d} z}=\hat{F}(\bar{\alpha}) \Phi(\tilde{y} ; \bar{\alpha}), \\
& \hat{u}_{2}=0 \quad \text { for } \quad z=z_{0}, \\
& \frac{\mathrm{d}^{2} \hat{u}_{2}}{\mathrm{~d} z^{2}}=(\mathrm{i} \bar{\alpha})^{1 / 3} \hat{p}_{2 w} \quad \text { for } \quad z=z_{0}, \\
& \hat{u}_{2}=\frac{\hat{p}_{2 w}}{|\bar{\alpha}|} \quad \text { for } \quad z=\infty,
\end{aligned}
$$

where $z=z_{0}+(\mathrm{i} \bar{\alpha})^{1 / 3} \tilde{y}$, with $z_{0}=(\mathrm{i} \bar{\omega}) /(\mathrm{i} \bar{\alpha})^{2 / 3}$, and

$$
\begin{aligned}
& \Phi(\tilde{y} ; \bar{\alpha})=(\mathrm{i} \bar{\alpha})^{-1} \frac{\mathrm{d}}{\mathrm{d} \tilde{y}}\left(\mathrm{i} \alpha U_{s}^{\circ} \Gamma+\Theta \frac{\mathrm{d} U_{s}^{\circ}}{\mathrm{d} \tilde{y}}\right), \\
& U_{s}^{\circ}=\frac{1}{2} \frac{\mu_{w}^{-1 / 4} \rho_{w}^{-1 / 2}}{\lambda^{1 / 4} \beta^{-1 / 4}} \frac{M}{1+M}\left[1-\mathrm{e}^{-(1+\mathrm{i}) \sqrt{\bar{\omega} / 2} \tilde{y}}\right] .
\end{aligned}
$$


Note that $(3.9 b)$ represents the Stokes layer disturbance $u_{s}$ evaluated at the centre of the roughness element. From equations (3.8) one can easily show that

$$
\hat{p}_{2 w}=\frac{|\bar{\alpha}| \operatorname{Ai}^{\prime}\left(z_{0}\right) \hat{F}(\bar{\alpha}) \int_{z_{0}}^{\infty} \eta(z) \mathrm{d} z}{\operatorname{Ai}^{\prime}\left(z_{0}\right)-(\mathrm{i} \bar{\alpha})^{1 / 3}|\bar{\alpha}| \int_{z_{0}}^{\infty} \operatorname{Ai}(z) \mathrm{d} z},
$$

where $\eta(z)$ is the solution of the boundary-value problem $\eta^{\prime \prime}-z \eta=\Phi$, with $\eta^{\prime}\left(z_{0}\right)=0$ and $\eta(\infty)=0$, and $\mathrm{Ai}$ and $\mathrm{Ai}^{\prime}$ are, respectively, the Airy function and its first derivative.

Finally, the wall pressure disturbance induced by the interaction between the acoustic wave and the roughness element can be obtained after taking the inverse Fourier transform, leading to

$$
\check{p}_{2 w}=-\sqrt{2 \pi} \mathcal{C}_{r} \hat{F}\left(\bar{\alpha}_{1}\right) \mathrm{e}^{\mathrm{i} \bar{\alpha}_{1} \tilde{x}} \text { for } \tilde{x} \rightarrow \infty,
$$

where

$$
\mathcal{C}_{r}=\left.\frac{\bar{\alpha} \operatorname{Ai}^{\prime}\left(z_{0}\right) \int_{z_{0}}^{\infty} \eta(z) \mathrm{d} z}{\frac{4}{3}(\mathrm{i} \bar{\alpha})^{1 / 3} \int_{z_{0}}^{\infty} \operatorname{Ai}(z) \mathrm{d} z-\frac{2}{3} \operatorname{Ai}\left(z_{0}\right) \frac{z_{0}}{\bar{\alpha}}\left[z_{0}+\mathrm{i}(\mathrm{i} \bar{\alpha})^{4 / 3}\right]}\right|_{\bar{\alpha}_{1}}
$$

is the receptivity coefficient. Here $\bar{\alpha}_{1}$ and $\bar{\omega}_{1}$ are, respectively, the wavenumber and frequency of a lower branch T-S wave. The inverse Fourier transform was calculated for $\bar{\omega} \rightarrow \bar{\omega}_{1}=2.29797$, in which case all the poles of (3.10) are complex with positive imaginary parts, except for the first one, $\bar{\alpha}_{1}=-1.0005$, which is real. Therefore, as $\tilde{x} \rightarrow \infty$ the contribution of the complex poles to the wall-pressure becomes negligible and the inverse Fourier transform may be easily calculated using the resiude of (3.10) at $\bar{\alpha}_{1}$. It is important to note that the expression at the denominator of equation (3.10) gives the well known large Reynolds number version of the Orr-Sommerfeld equation for a Blasius boundary layer, i.e.

$$
\operatorname{Ai}^{\prime}\left(z_{0}\right)-(\mathrm{i} \bar{\alpha})^{1 / 3}|\bar{\alpha}| \int_{z_{0}}^{\infty} \operatorname{Ai}(z) \mathrm{d} z=0,
$$

whose first root describes the T-S wave.

\subsection{The vibrating ribbon problem}

The shape of the vibrating ribbon may be written using lower-deck coordinates as $\bar{y}_{v}=$ $\epsilon \bar{F}(\bar{x}) G(\bar{t})$. In this case, we look for solutions to (3.3)-(3.4) in the following form

$$
\bar{U}=\bar{y}+\epsilon u_{1}+\cdots, \quad \bar{V}=\epsilon v_{1}+\cdots, \quad \bar{P}=\epsilon p_{1}+\cdots .
$$

In order to model the presence of a vibrating ribbon we use slip velocities at $\bar{y}=0$. These are obtained by expanding the near wall flow in Taylor series and retaining the dominant terms; we obtain $u_{1}=-\bar{F}(\bar{x}) G(\bar{t})$ and $v_{1}=\bar{F}(\bar{x}) G^{\prime}(\bar{t})$ at $\bar{y}=0$.

Since the vibrating ribbon introduces time-harmonic oscillations into the system, the problem admits time-harmonic solutions $u_{1}=1 / 2 \check{u}_{1} \exp (\mathrm{i} \bar{\omega} \bar{t})+$ c.c.. It can then be shown 
that, in Fourier space, equations (3.3)-(3.4) reduce to

$$
\begin{aligned}
& \frac{\mathrm{d}^{3} \hat{u}_{1}}{\mathrm{~d} z^{3}}-z \frac{\mathrm{d} \hat{u}_{1}}{\mathrm{~d} z}=0, \\
& \hat{u}_{1}=-\hat{F}(\bar{\alpha}) \quad \text { for } \quad z=z_{0}, \\
& \frac{\mathrm{d}^{2} \hat{u}_{1}}{\mathrm{~d} z^{2}}=(\mathrm{i} \bar{\alpha})^{1 / 3} \hat{p}_{1 w} \quad \text { for } \quad z=z_{0}, \\
& \hat{u}_{1}=\frac{\hat{p}_{1 w}}{|\bar{\alpha}|} \quad \text { for } \quad z=\infty .
\end{aligned}
$$

From the above equations one can easily find that

$$
\hat{p}_{1 w}=-\frac{|\bar{\alpha}| \operatorname{Ai}^{\prime}\left(z_{0}\right) \hat{F}(\bar{\alpha})}{\operatorname{Ai}^{\prime}\left(z_{0}\right)-(\mathrm{i} \bar{\alpha})^{1 / 3}|\bar{\alpha}| \int_{z_{0}}^{\infty} \operatorname{Ai}(z) \mathrm{d} z} .
$$

The wall pressure disturbance introduced by the vibrating ribbon is finally obtained by taking the inverse Fourier transform, leading to

$$
\check{p}_{1 w}=\sqrt{2 \pi} \mathcal{C}_{v} \hat{F}\left(\bar{\alpha}_{1}\right) \mathrm{e}^{\mathrm{i} \bar{\alpha}_{1} \bar{x}} \text { for } \bar{x} \rightarrow \infty,
$$

where

$$
\mathcal{C}_{v}=\left.\frac{\bar{\alpha} \operatorname{Ai}^{\prime}\left(z_{0}\right)}{\frac{4}{3}(\mathrm{i} \bar{\alpha})^{1 / 3} \int_{z_{0}}^{\infty} \operatorname{Ai}(z) \mathrm{d} z-\frac{2}{3} \operatorname{Ai}\left(z_{0}\right) \frac{z_{0}}{\bar{\alpha}}\left[z_{0}+\mathrm{i}(\mathrm{i} \bar{\alpha})^{4 / 3}\right]}\right|_{\bar{\alpha}_{1}}
$$

is the receptivity coefficient.

\section{Details of the numerical study}

4.1. The governing equations and their numerical treatment

The receptivity problems discussed theoretically in the previous sections may also be analysed numerically by directly solving the compressible Navier-Stokes equations. In this work a generic flow variable $\psi$ is decomposed into a steady part $\bar{\psi}$ and an unsteady part $\psi^{\prime}$. The steady part of the flow is calculated using the SBLI code, developed at the University of Southampton, which solves the full compressible Navier-Stokes equations written in dimensionless form as

$$
\begin{aligned}
& \frac{\partial \bar{\rho}}{\partial t}+\frac{\partial \bar{\rho} \bar{u}_{j}}{\partial x_{j}}=0, \\
& \frac{\partial \bar{\rho} \bar{u}_{i}}{\partial t}+\frac{\partial \bar{\rho} \bar{u}_{i} \bar{u}_{j}}{\partial x_{j}}+\frac{\partial \bar{p}}{\partial x_{i}}=\frac{\partial \bar{\tau}_{i j}}{\partial x_{j}}, \\
& \frac{\partial \bar{\rho} \bar{E}}{\partial t}+\frac{\partial(\bar{\rho} \bar{E}+\bar{p}) \bar{u}_{i}}{\partial x_{i}}=-\frac{\partial \bar{q}_{i}}{\partial x_{i}}+\frac{\partial \bar{u}_{i} \bar{\tau}_{i j}}{\partial x_{j}} .
\end{aligned}
$$

These are advanced in time until convergence starting from a suitable initial condition. The components $\bar{\tau}_{i j}$ of the viscous stress tensor are defined as

$$
\bar{\tau}_{i j}=\frac{\bar{\mu}}{R e}\left(\frac{\partial \bar{u}_{j}}{\partial x_{i}}+\frac{\partial \bar{u}_{i}}{\partial x_{j}}-\frac{2}{3} \frac{\partial \bar{u}_{k}}{\partial x_{k}} \delta_{i j}\right),
$$


where $\delta_{i j}$ is the usual Kronecker delta function. The total energy per unit mass is defined as

$$
\bar{E}=\frac{\bar{T}}{\gamma(\gamma-1) M^{2}}+\frac{1}{2} \bar{u}_{i} \bar{u}_{i}
$$

and the dynamic viscosity is calculated from the temperature field using the Sutherland's law $\bar{\mu}=\bar{T}^{3 / 2}\left(1+S^{*} / T_{\infty}^{*}\right) /\left(\bar{T}+S^{*} / T_{\infty}^{*}\right)$, where $S^{*}=110.4 \mathrm{~K}$ is the Sutherland constant for air and $T_{\infty}^{*}=273.15 \mathrm{~K}$. The pressure $\bar{p}$ and the heat fluxes $\bar{q}_{i}$ are calculated from the equation of state and Fourier's law of heat conduction, given respectively by

$$
\bar{p}=\frac{\bar{\rho} \bar{T}}{\gamma M^{2}} \quad \text { and } \quad \bar{q}_{i}=-\frac{\bar{\mu}}{(\gamma-1) M^{2} \operatorname{Pr} \operatorname{Re}} \frac{\partial \bar{T}}{\partial x_{i}} .
$$

Having calculated the basic flow, the same equations may in principle be solved to obtain the unsteady part of the flow. However, since in this work the unsteady perturbations are considered to be small, it was convenient to linearise the equations about the steady flow and adapt the SBLI code to solve the linearised compressible Navier-Stokes equations, written in the following form

$$
\begin{aligned}
& \frac{\partial \rho^{\prime}}{\partial t}+\frac{\partial \bar{\rho} u_{i}^{\prime}}{\partial x_{i}}+\frac{\partial \rho^{\prime} \bar{u}_{i}}{\partial x_{i}}=0 \\
& \frac{\partial u_{i}^{\prime}}{\partial t}+\left(\frac{\rho^{\prime}}{\bar{\rho}} \bar{u}_{j}+u_{j}^{\prime}\right) \frac{\partial \bar{u}_{i}}{\partial x_{j}}+\bar{u}_{j} \frac{\partial u_{i}^{\prime}}{\partial x_{j}}+\frac{1}{\bar{\rho}} \frac{\partial p^{\prime}}{\partial x_{i}}=\frac{1}{\bar{\rho}} \frac{\partial \tau_{i j}^{\prime}}{\partial x_{j}} \\
& \frac{\partial T^{\prime}}{\partial t}+\bar{u}_{i} \frac{\partial T^{\prime}}{\partial x_{i}}+\left(\frac{\rho^{\prime}}{\bar{\rho}} \bar{u}_{i}+u_{i}^{\prime}\right) \frac{\partial \bar{T}}{\partial x_{i}}+\mathcal{B}\left(\bar{p} \frac{\partial u_{i}^{\prime}}{\partial x_{i}}+p^{\prime} \frac{\partial \bar{u}_{i}}{\partial x_{i}}\right)=-\mathcal{B} \frac{\partial q_{i}^{\prime}}{\partial x_{i}}+\mathcal{B D}^{\prime},
\end{aligned}
$$

where $\mathcal{B}=\gamma(\gamma-1) M^{2} / \bar{\rho}$. The unsteady pressure $p^{\prime}$ and the linearised heat fluxes $q_{i}^{\prime}$ are given by the linearised versions of equations (4.4) and may be written respectively as

$$
p^{\prime}=\frac{1}{\gamma M^{2}}\left(\bar{\rho} T^{\prime}+\rho^{\prime} \bar{T}\right) \quad \text { and } \quad q_{i}^{\prime}=-\frac{1}{(\gamma-1) M^{2} \operatorname{PrRe}}\left(\bar{\mu} \frac{\partial T^{\prime}}{\partial x_{i}}+\mu^{\prime} \frac{\partial \bar{T}}{\partial x_{i}}\right),
$$

while the components $\tau_{i j}^{\prime}$ of the linear viscous stress tensor appearing in $(4.5 b)$ and the linear viscous dissipation term $\mathcal{D}^{\prime}$ appearing in $(4.5 c)$ are given respectively by

$$
\tau_{i j}^{\prime}=\frac{1}{R e}\left\{\bar{\mu}\left(\frac{\partial u_{i}^{\prime}}{\partial x_{j}}+\frac{\partial u_{j}^{\prime}}{\partial x_{i}}-\frac{2}{3} \frac{\partial u_{k}^{\prime}}{\partial x_{k}} \delta_{i j}\right)+\mu^{\prime}\left(\frac{\partial \bar{u}_{i}}{\partial x_{j}}+\frac{\partial \bar{u}_{j}}{\partial x_{i}}-\frac{2}{3} \frac{\partial \bar{u}_{k}}{\partial x_{k}} \delta_{i j}\right)\right\}
$$

and

$$
\mathcal{D}^{\prime}=\frac{\partial \bar{u}_{i}}{\partial x_{j}} \tau_{i j}^{\prime}+\frac{\partial u_{i}^{\prime}}{\partial x_{j}} \bar{\tau}_{i j}
$$

Note that, since here we are interested in the two-dimensional problem, the summation indexes $i, j$ and $k$ take values 1 and 2 and we put $\left(x_{1}, x_{2}\right)=(x, y)$ and $\left(u_{1}, u_{2}\right)=(u, v)$.

Both the full and the linearised compressible Navier-Stokes equations are solved numerically for generalised curvilinear coordinates using high order finite-differences. The spatial discretisation is treated using a standard fourth-order central difference scheme to calculate derivatives at internal points, while close to boundaries a stable boundary treatment by Carpenter et al. (1999) is applied, giving overall fourth-order accuracy. Time integration is based on a third-order compact Runge-Kutta method (Wray 1990). The full nonlinear code employs an entropy splitting approach developed by Sandham et al. (2002), whereby the inviscid flux derivatives are split into conservative and nonconservative parts. The entropy splitting scheme, together with a Laplacian formulation 


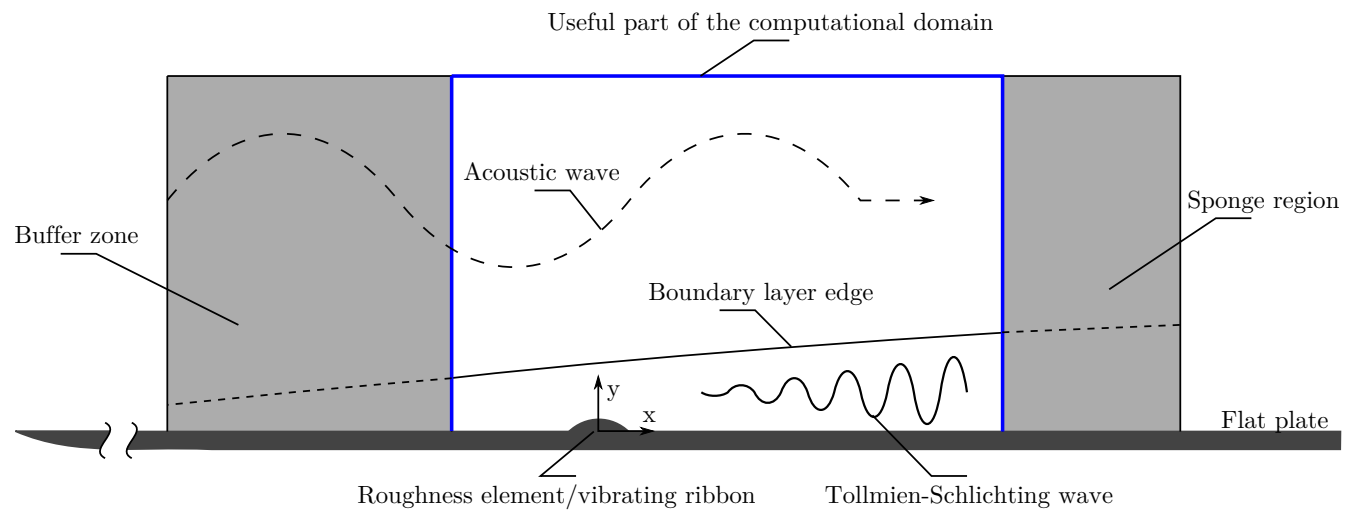

FiguRE 3. Schematic representation of the numerical treatment, showing the buffer zone and the sponge region in grey, which are place respectively upstream and downstream of the useful par of the computational domain.

of the heat transfer and viscous dissipation terms in the momentum (4.1b) and energy (4.1c) equations (which prevents the odd-even decoupling typical of central differences, see Sandham et al. 2002), helps improve the stability of the low dissipative spatial discretisation scheme used. The linearised code retains the Laplacian formulation for the heat transfer term in the momentum (4.5b) and energy (4.5c) equations and for the viscous dissipation term in the momentum equation. The SBLI code has multi-block capabilities and is made parallel (both intra- and inter-block) using the Message Passing Interface (MPI) library. The code has been extensively validated (see for example De Tullio 2013; De Tullio \& Sandham 2010; De Tullio et al. 2013).

\subsection{Flow configurations, computational domains and boundary conditions}

A schematic representation of the numerical technique used for the receptivity studies is given in figure 3. The numerical simulations are performed on a computational domain with the inflow boundary starting downstream of the flat plate leading edge. In the case of the nonlinear Navier-Stokes simulations, the domain inflow is initialised with a compressible laminar similarity solution obtained after numerically solving (2.3) and a pressure extrapolation boundary condition is then applied, whereby the inflow conservative variables are calculated by linearly extrapolating the pressure from within the domain. Wave reflections from the domain external boundaries are controlled through the use of characteristic boundary conditions (originally derived by Thomson 1987, 1990) at the top and outflow boundaries. In addition, a sponge region is introduced near the outflow boundary, where at the end of each time-step the conservative variables vector $\mathbf{q}$ is updated as $\mathbf{q}_{\text {new }}=\mathbf{q}_{\text {old }}-\sigma \Delta t / 2\left[1+\cos \left(\pi \xi / L_{s p}\right)\right]\left\{\mathbf{q}_{\text {old }}-\mathbf{q}_{\text {ref }}\right\}$, where $\Delta t$ is the simulation time-step, $\sigma=0.05$ is a damping factor, $\xi$ is the streamwise coordinate measured from the start of the sponge, $L_{s p}$ is the streamwise extent of the sponge and $\mathbf{q}_{r e f}$ is given by the similarity solution vector used for the flow initialisation. The characteristic conditions and the sponge region are used in the nonlinear Navier-Stokes simulations to minimize transients to steady state. No-slip and isothermal boundary conditions are applied at the wall, where the wall-temperature is given by equation (2.5).

The linearised Navier-Stokes equations are solved using characteristic boundary conditions for the top and outflow boundaries in all cases and also for the inflow boundary for the cases involving a vibrating ribbon. The walls are considered no-slip and isothermal. For the study of the receptivity due to sound, an accurate formulation of the inflow boundary conditions is of critical importance. In this case a prescribed time-varying in- 
flow boundary condition is used, whereby the acoustic waves are introduced using the complex vector $\boldsymbol{\psi}$ of eigenfunctions of the downstream travelling acoustic modes, which is obtained from parallel compressible linear stability computations (see Balakumar \& Malik 1992). The boundary condition reads $\mathbf{q}_{0}=\boldsymbol{\psi} \exp (\mathrm{i} 2 \pi f t)+$ c.c., where $f$ is a dimensionless frequency. It is important to note that, since the inflow condition used for the nonlinear Navier-Stokes simulations is not a solution of the full Navier-Stokes equations, a small region is present near the domain inflow where the basic flow undergoes a slight adjustment. The coupling between the unsteady acoustic disturbances imposed at the inflow and the adjustment of the basic flow leads to the excitation of a small T-S wave immediately downstream of the inflow boundary, which in turn affects the acoustic receptivity process at the roughness location. Therefore, in order to obtain a clean disturbance field, this small region is removed from the basic flow used in the linearised Navier-Stokes simulations and a buffer region is introduced near the inflow boundary where any residual numerical oscillations induced due to the introduction of the acoustic mode eigenfunctions are eliminated by a combination of filtering and grid stretching. As will be shown in $\S 5.1$, this technique leads to a disturbance field given only by an acoustic wave travelling in the flow direction and the induced Stokes layer.

The vibrating ribbon receptivity study is carried out using the laminar boundary layer over the smooth flat plate as the basic flow and the vibrating ribbon is modelled in the linearised Navier-Stokes simulations by appropriate slip velocities at the flat plate wall. Similarly to the approach used for the derivation of the theoretical result, the slip velocities, which are needed in order to satisfy the no-slip condition at the surface of the vibrating ribbon, are calculated by Taylor expanding the boundary layer flow around $y=0$. After retaining only the principal terms in the Taylor expansion we obtain

$$
u_{w}^{\prime}=-\frac{\partial \bar{u}}{\partial y} F(x) G(t), \quad v_{w}^{\prime}=F(x) \frac{\mathrm{d} G(t)}{\mathrm{d} t},
$$

where $G(t)=\cos (2 \pi f t)$. The linearised Navier-Stokes simulations employ a sponge region (with $\mathbf{q}_{r e f}=\mathbf{0}$ ) near the outflow boundary to absorb the downstream travelling waves (acoustic and T-S waves). The buffer region is used in all cases, as it also damps the upstream travelling acoustic waves which may be scattered by the roughness or the vibrating ribbon. Of course, the results obtained in the buffer and sponge regions are discarded and only the results obtained in the remaining useful part of the computational domain will be considered in the analysis.

The roughness element and the vibrating ribbon are assumed to have the same Gaussian shape given by

$$
F(x)=\exp \left[-\frac{x^{2}}{2(\Delta / 6)^{2}}\right],
$$

where $\Delta$ is used as a dimensionless measure of the width of the Gaussian; it gives approximately the full Gaussian width at one percent of its maximum. The flow over the roughness element was calculated by solving the nonlinear Navier-Stokes equations using a body-fitted computational grid. A roughness height of $h^{*} / \delta_{i n}^{*}=10^{-3}$ was considered in all cases, where $\delta_{i n}^{*}$ is the displacement thickness evaluated at the start of the useful part of the computational domain. Note that, since the unsteady flows are calculated by solving the linearised Navier-Stokes equations, the amplitudes of the vibrations of the ribbon and the acoustic waves are not relevant for the analysis.

The main part of the numerical study focuses on the assessment of the asymptotic theories for the prediction of the linear receptivity of lower branch T-S waves. To this end, 


$\begin{array}{lcccccccc}\text { Case } & M & T_{w}^{*} / T_{\infty}^{*} & \mathcal{F}^{\prime \prime}(0) & R e & R e_{\delta_{i n}^{*}} & \Delta^{*} / \delta_{i n}^{*} & \Delta / \lambda_{T S} & f=f^{*} \mu_{\infty}^{*} / \rho_{\infty}^{*} U_{\infty}^{*} \\ \text { M0.2A1 } & 0.2 & 1.007 & 0.4700 & 35.1 \times 10^{6} & 10000 & 100.0 & 1.0 & 1.860 \times 10^{-7} \\ \text { M0.2A2 } & 0.2 & 1.007 & 0.4700 & 35.1 \times 10^{6} & 10000 & 50.0 & 0.50 & 1.860 \times 10^{-7} \\ \text { M0.2A3 } & 0.2 & 1.007 & 0.4700 & 35.1 \times 10^{6} & 10000 & 75.0 & 0.75 & 1.860 \times 10^{-7} \\ \text { M0.2A4 } & 0.2 & 1.007 & 0.4700 & 35.1 \times 10^{6} & 10000 & 125.0 & 1.25 & 1.860 \times 10^{-7} \\ \text { M0.2A5 } & 0.2 & 1.007 & 0.4700 & 35.1 \times 10^{6} & 10000 & 150.0 & 1.50 & 1.860 \times 10^{-7} \\ \text { M0.2B } & 0.2 & 1.007 & 0.4700 & 8.4 \times 10^{6} & 4750 & 80.0 & 1.0 & 5.747 \times 10^{-7} \\ \text { M0.2C } & 0.2 & 1.007 & 0.4700 & 4.8 \times 10^{6} & 3500 & 73.25 & 1.0 & 9.143 \times 10^{-7} \\ \text { M0.2D } & 0.2 & 1.007 & 0.4700 & 2.6 \times 10^{6} & 2500 & 66.71 & 1.0 & 1.512 \times 10^{-6} \\ \text { M0.2E } & 0.2 & 1.007 & 0.4700 & 1.0 \times 10^{6} & 1500 & 58.27 & 1.0 & 3.065 \times 10^{-6} \\ \text { M0.2F } & 0.2 & 1.007 & 0.4700 & 0.5 \times 10^{6} & 965 & 55.97 & 1.0 & 5.637 \times 10^{-6} \\ \text { M0.2G } & 0.2 & 1.007 & 0.4700 & 0.1 \times 10^{6} & 325 & 40.85 & 1.0 & 2.991 \times 10^{-5} \\ \text { M0.4A } & 0.4 & 1.027 & 0.4713 & 35.1 \times 10^{6} & 10280 & 103.3 & 1.0 & 5.536 \times 10^{-7} \\ \text { M0.4B } & 0.4 & 1.027 & 0.4713 & 8.4 \times 10^{6} & 4877 & 82.71 & 1.0 & 1.780 \times 10^{-7} \\ \text { M0.6A } & 0.6 & 1.061 & 0.4735 & 35.1 \times 10^{6} & 10740 & 110.2 & 1.0 & 5.149 \times 10^{-7} \\ \text { M0.6B } & 0.6 & 1.061 & 0.4735 & 8.4 \times 10^{6} & 5088 & 87.95 & 1.0 & 1.653 \times 10^{-7} \\ \text { M0.8A } & 0.8 & 1.108 & 0.4766 & 35.1 \times 10^{6} & 11370 & 122.0 & 1.0 & 4.649 \times 10^{-7} \\ \text { M0.8B } & 0.8 & 1.108 & 0.4766 & 8.4 \times 10^{6} & 5377 & 96.52 & 1.0 & 1.482 \times 10^{-7}\end{array}$

TABLE 1. Details of the numerical simulations performed. Each case shows the numerical simulation parameters used for both the acoustic wave and vibrating ribbon receptivity studies. Note that here $\Delta^{*}$ is the dimensional version of $\Delta$ and $\lambda_{T S}$ is the dimensionless T-S wave wavelength and $f^{*}$ is dimensional frequency measured in cycles per second.

a parametric study is carried out, where the modifications introduced by the variations of the roughness element or vibrating ribbon longitudinal dimension $\Delta$, the Reynolds number and the Mach number are analysed and compared with the theoretical predictions. Details of the numerical simulations carried out are given in table 1 . It should be noted that each of the cases shown in the table refers to both the acoustic wave and vibrating ribbon receptivity studies. For the acoustic wave receptivity study two nonlinear Navier-Stokes and two linearised Navier-Stokes simulations are performed for each case to obtain, respectively, the basic flows and the unsteady fields for the cases with and without roughness element. On the other hand, for the vibrating ribbon receptivity study only one nonlinear and one linearised Navier-Stokes simulations are required per case. The frequency of the unsteady perturbations introduced in each case corresponds to the frequency of a T-S wave on the lower branch of the neutral stability curve at $x=0$ and are given in table 1 . Additional numerical simulations have been performed to investigate the effects of T-S wave frequency and roughness height, the details of which are reported in $\S 5.3$.

When normalised by $\delta_{i n}^{*}$, the size of the computational domains used is the same in all cases. The streamwise and wall-normal extents of the useful computational domain portion are $L_{x}^{*} / \delta_{i n}^{*} \times L_{y}^{*} / \delta_{i n}^{*}=550 \times 160$ and the number of grid-points in the $x$ and $y$ directions are $N_{x} \times N_{y}=501 \times 415$. Note that normalisation by $L^{*}$ may be easily recovered by making use of the Reynolds numbers $R e$ (based on $L^{*}$ ) and $R e_{\delta_{i n}^{*}}$ (based on $\delta_{i n}^{*}$ ) given in table 1 . The numerical grid employed to obtain the results presented in the following has constant grid spacing in the streamwise direction and is stretched in the wall-normal direction according to $y=L_{y} \sinh \left(b_{y} \eta\right) / b_{y}$, where $0 \leqslant \eta \leqslant 1$ and $b_{y}=5.342$ is the stretching factor; it was chosen after a grid convergence study which showed negligible variations of the receptivity and subsequent linear growth of the T-S waves when using a grid with double the number of points in each direction. 


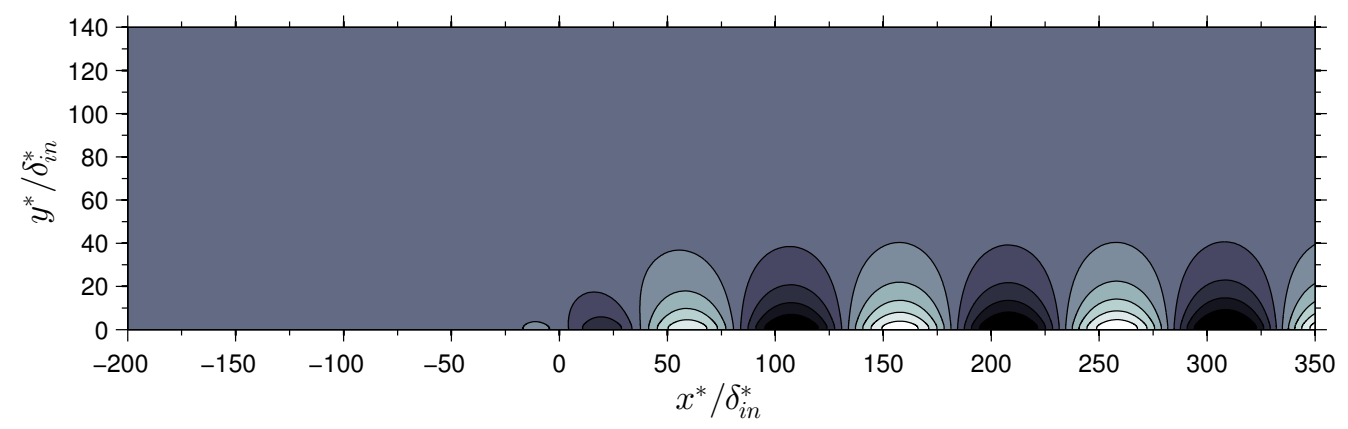

FiguRE 4. Boundary layer response due to the interaction of an acoustic wave with an isolated roughness element for case M0.2A1. The plot shows contours of instantaneous pressure disturbance $p_{2}$.

\section{Numerical results and comparisons with theory}

\subsection{Lower branch $T$-S wave receptivity by sound}

The T-S wave receptivity by sound is analysed numerically by subjecting the laminar basic flow over the roughness element to the inflow disturbances described in $\S 4.2$. Here, for consistency with the terminology used for the derivation of the theoretical result, the solution obtained for any flow variable, say the $u$-velocity, is decomposed as

$$
u=U_{B}+u_{s}+u_{1}+u_{2},
$$

where $U_{B}$ represents the boundary layer flow over a smooth flat plate, $u_{s}$ is the unsteady disturbance introduced by the acoustic wave, $u_{1}$ is the steady disturbance introduced by the roughness element and $u_{2}$ is the disturbance generated by the interaction between $u_{s}$ and $u_{1}$. The last term includes the evolution of the T-S waves and is obtained as the difference between the linearised Navier-Stokes results obtained for the cases with and without roughness element.

An example of the pressure disturbance $p_{2}$ induced in the boundary layer by the interaction between the acoustic wave and the roughness element at $M=0.2$ is shown in figure 4. It can be seen that the interaction provides the frequency and wavenumber resonance conditions required for the excitation of a T-S wave in the boundary layer downstream of the roughness element. The acoustic wave/roughness element interaction also leads to the scattering of an additional acoustic wave. At $M=0.2$ the amplitude of this wave is small, hence is not visible in figure 4, but it grows as the Mach number increases.

Before attempting a comparison between the numerical and theoretical results a verification of the numerical solutions obtained is in order. In particular, it is important to verify that the disturbances imposed at the inflow boundary introduce "clean" acoustic waves (and the induced Stokes layer) in the useful part of the computational domain (i.e. any residual numerical disturbances triggered at the inflow of the computational domain are successfully dissipated within the buffer region) and that the disturbances generated by the acoustic wave/roughness element interaction are well captured by the numerical scheme. Figure 5(a) shows the $u_{s}$-disturbance amplitude profile (normalised with the amplitude of the pressure perturbations in the free stream) obtained at $x=0$ in the case of a smooth flat plate for the different Mach numbers considered. The Navier-Stokes solutions are in excellent agreement with the acoustic mode eigenfunctions obtained from linear stability theory, indicating that the disturbances introduced in the numerical simulations are a close representation of the disturbances induced by a plane acoustic wave travel- 
(a)

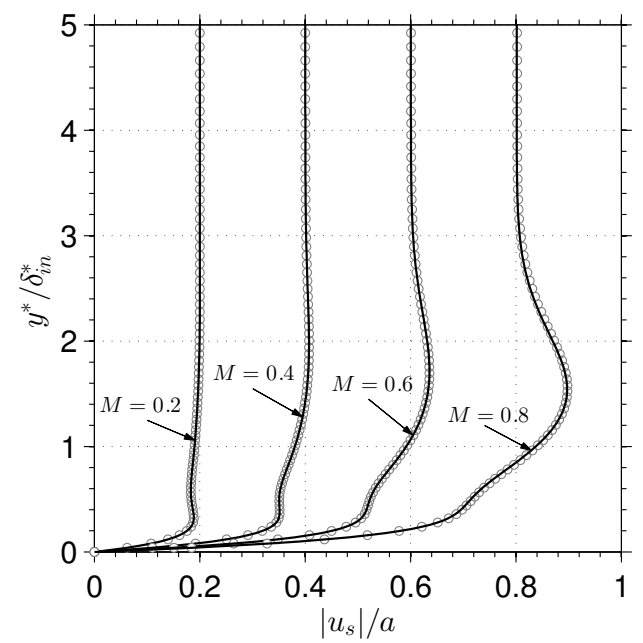

Nicola De Tullio, Anatoly I. Ruban

(b)

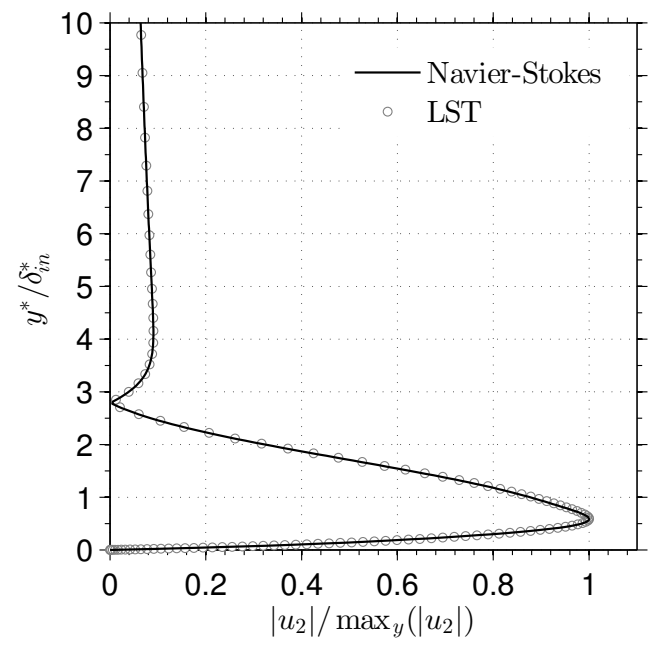

FIGURE 5. Comparing the linearised Navier-Stokes results with linear stability calculations. (a) Disturbances induced in the boundary layer by the acoustic wave in the absence of a roughness element for cases M0.2A1, M0.4A, M0.6A and M0.8A. (b) The $u$-velocity amplitude function at $x=340$ of the T-S wave generated by the interaction between the acoustic wave and the roughness element for case M0.2A1.

ling in the flow direction. Figure $5(\mathrm{~b})$ gives a comparison between the $u_{2}$-disturbance amplitude profile obtained for case M0.2A1 at $x=340$ and the corresponding T-S wave eigenfunction obtained from LST at the same $x$-position. The excellent agreement indicates that the interaction between the acoustic waves and the roughness element leads to the excitation of a T-S wave (in addition to other stable waves) which is well captured in the numerical solution.

The comparisons between the asymptotic theory and the Navier-Stokes simulations are performed here for the wall-pressure disturbance $p_{2 w}$ induced by the interaction between the acoustic wave and the roughness element. The amplitude distribution of $p_{2 w}$ along the streamwise direction is extracted by projecting the numerical results into Fourier space at each $x$-position using the following discrete Fourier transform formula

$$
A_{p_{w}}(x)=\frac{2}{N}\left|\sum_{j=1}^{N} \tilde{p}_{w}\left(x, t_{j}\right) \mathrm{e}^{-\mathrm{i} \frac{2 \pi(j-1)}{N}}\right|,
$$

where $\mathrm{i}$ is the complex unity and $N$ is the total number of samples taken over one period of the disturbance signal. Notice that only one frequency is excited in each of the cases analysed, hence there is only one non-zero Fourier coefficient. The normalisation factor $2 / N$ is necessary to recover the disturbance amplitude in the physical space; it gives unit Fourier coefficients for a disturbance signal given by sinusoidal waves. The results are shown in figures 6(a) and 6(b) (black lines) for the different Reynolds numbers and Mach numbers considered, respectively. The figures also show the T-S wave growth predicted using local spatial LST by calculating the growth rate $-\alpha_{\mathrm{i}}$ at different $x$-positions and integrating in the streamwise direction. It can be seen that, after an initial beating, the wall-pressure disturbance signal grows monotonically in the downstream direction following the predicted T-S wave behaviour. Note that the numerical simulations were designed to excite a T-S wave on the lower branch of the neutral stability curve at the 
(a)

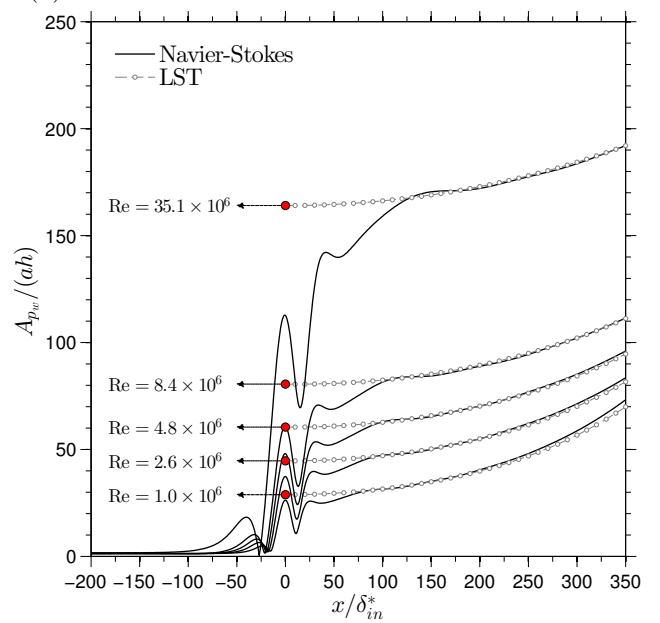

(b)

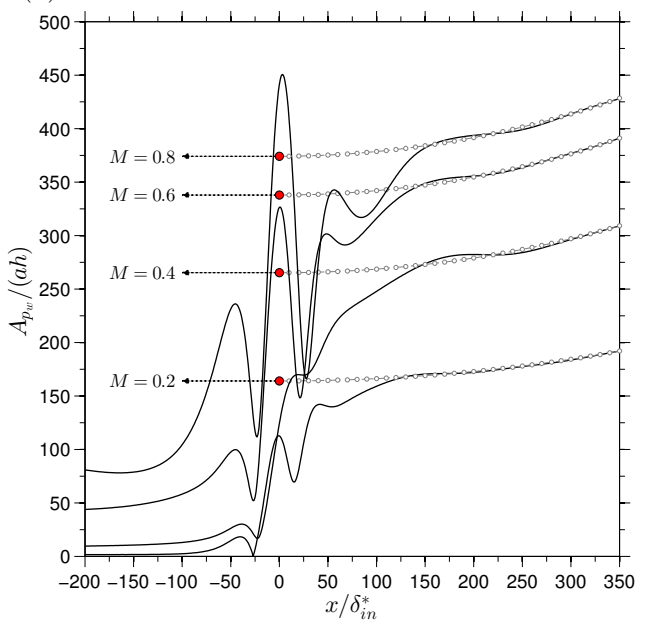

FIGURE 6. Streamwise variation of the amplitude wall-pressure perturbation obtained in the presence of a roughness element: (a) cases M0.2A1, M0.2B, M0.2C, M0.2D and M0.2E; (b) cases M0.2A1, M0.4A, M0.6A and M0.8A.

centre of the roughness element. In fact, based on LST, the growth rate is zero at $x=0$ and increases further downstream as the wave enters the unstable region of the boundary layer.

In this work we are interested in the initial amplitude of the T-S wave generated by the interaction. In the vicinity of the roughness, the perturbation field is rather complicated. However, downstream of the roughness only one perturbation mode survives, the T$\mathrm{S}$ wave. Our task is to find the initial amplitude of this wave, which is recovered by making use of the LST result; the amplitude growth predicted by LST is scaled to match the Navier-Stokes solution downstream of the initial beating, in a region where the disturbance signal is dominated by the T-S wave, say $x=x_{p}$. The amplitude $A_{0}$ of the wave at the centre of the roughness element is then simply given by the scaled LST result at $x=0$, shown by the grey-filled circles (red online) in figure 6 . It is calculated as

$$
A_{0}=\frac{A_{p_{w}}\left(x_{p}\right)}{\exp \left[\int_{0}^{x_{p}}-\alpha_{\mathrm{i}}(x) \mathrm{d} x\right]} .
$$

The initial T-S wave amplitudes extracted from the Navier-Stokes solutions for the different flow conditions considered are compared with the theoretical predictions in figure 7 . Since the problem is linear, the T-S wave amplitude $A_{0}$ is normalised by the acoustic wave amplitude $a$ and the roughness height $h$. In order to compare the numerical results with theory we first note that the theoretical results presented in $\S 3.1$ imply that

$$
\begin{aligned}
& p_{2 w}=\epsilon \delta R e^{-1 / 4} \frac{\mu_{w}^{1 / 2}}{\lambda^{-1 / 2} \beta^{1 / 2}}\left\{\frac{1}{2} \check{p}_{2 w} \mathrm{e}^{\mathrm{i} \bar{\omega} \tilde{t}}+\text { c.c. }\right\}, \\
& \check{p}_{2 w}=-\sqrt{2 \pi} \mathcal{C}_{r} \hat{F}\left(\bar{\alpha}_{1}\right) \mathrm{e}^{\mathrm{i} \bar{\alpha}_{1} \tilde{x}}
\end{aligned}
$$

where the receptivity coefficient $\mathcal{C}_{r}$ is given by (3.12) and, based on equation (4.10), we 

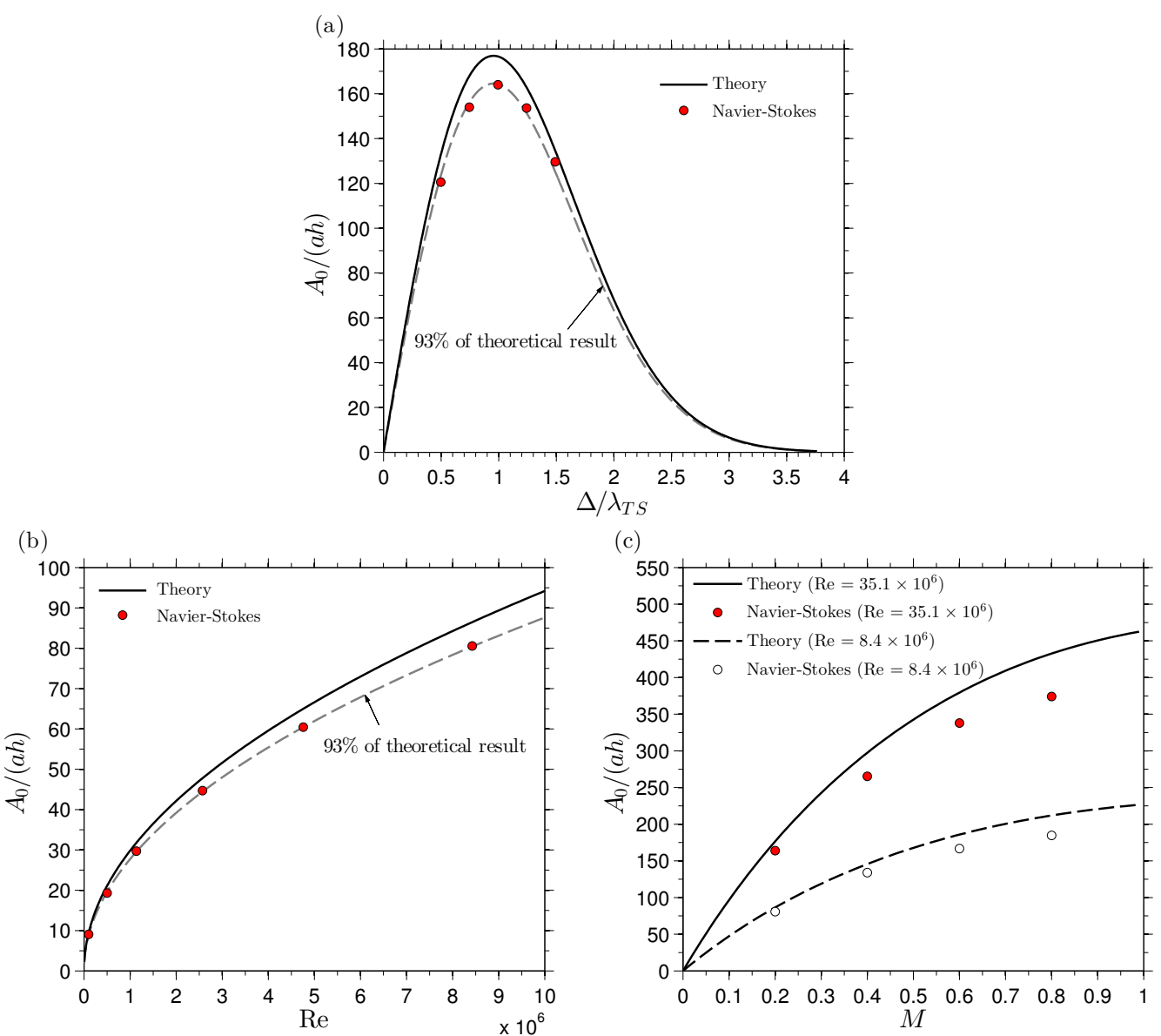

FiguRE 7. Comparisons between the asymptotic theory and the Navier-Stokes simulations for the acoustic wave receptivity study, showing the variation of initial T-S wave amplitude as a function of: (a) $\Delta / \lambda_{T S}$ for $R e=35.1 \times 10^{6}$ and $M=0.2$ (numerical cases M0.2A1, M0.2A2, $\mathrm{M} 0.2 \mathrm{~A} 3, \mathrm{M} 0.2 \mathrm{~A} 4$ and M0.2A5); (b) $R e$ for $M=0.2$ and $\Delta / \lambda_{T S}=1$ (numerical cases M0.2B, $\mathrm{M} 0.2 \mathrm{C}, \mathrm{M} 0.2 \mathrm{D}, \mathrm{M} 0.2 \mathrm{E}, \mathrm{M} 0.2 \mathrm{~F}$ and $\mathrm{M} 0.2 \mathrm{G}$ ); (c) $M$ for $\Delta / \lambda_{T S}=1$ and $R e=35.1 \times 10^{6}$ and $R e=8.4 \times 10^{6}$ (numerical cases M0.2A1, M0.2B, M0.4A, M0.4B, M0.6A, M0.6B, M0.8A and $\mathrm{M} 0.8 \mathrm{~B})$

may write

$$
\hat{F}\left(\bar{\alpha}_{1}\right)=\frac{\mu_{w}^{-1 / 4} \rho_{w}^{1 / 2}}{\lambda^{-3 / 4} \beta^{-1 / 4}} \frac{2 \pi}{6 \bar{\alpha}_{1}} \frac{\Delta}{\lambda_{T S}} \exp \left[-\frac{\pi^{2}}{18}\left(\frac{\Delta}{\lambda_{T S}}\right)^{2}\right],
$$

where $\lambda_{T S}$ is the dimensionless T-S wave wavelength. Finally, the initial T-S wave amplitude predicted by the asymptotic theory is given by

$$
\frac{A_{0}}{a h}=\underbrace{R e^{5 / 8}}_{\epsilon / h} \underbrace{R e^{1 / 8}}_{\delta / a} R e^{-1 / 4} \frac{\mu_{w}^{1 / 2}}{\lambda^{-1 / 2} \beta^{1 / 2}}\left|\check{p}_{2 w}\right| .
$$

Figure 7(a) shows the initial T-S wave amplitude as a function of roughness longitudinal dimension $(\Delta)$ to T-S wavelength $\left(\lambda_{T S}\right)$ ratio for $R e=35.1 \times 10^{6}$ and $M=0.2$. The numerical results are in excellent agreement with the theory, which in turn shows that, for fixed $R e, M$ and $T_{w}$, the T-S wave receptivity is only a function of the roughness element 
Fourier coefficient corresponding to the T-S wave wavenumber, rather than being directly affected by the shape of the roughness element. It is easily shown that, theoretically, the maximum receptivity is obtained for $\Delta / \lambda_{T S}=3 / \pi$, which is in good agreement with the numerical results. Figure 7(b) gives the variation of initial T-S wave amplitude as a function of Reynolds number, for $M=0.2$ and $\Delta / \lambda_{T S}=1$. Both the theory (see 5.6) and the Navier-Stokes simulations show that $A_{0} /(a h) \propto R e^{1 / 2}$. It is interesting to note that the behaviour predicted by the asymptotic theory for large values of the Reynolds number seems to be maintained also at low Reynolds numbers. In fact, the relative error between theory and Navier-Stokes simulations remains approximately constant and roughly equal to $7 \%$ of the theoretical result across the whole Reynolds number range considered. Figure 7(c) shows that the agreement remains good as the Mach number is increased, albeit the relative error increases slightly with Mach number to reach a maximum of about $13.5 \%$ at $M=0.8$. The asymptotic theory indicates that, when $R e$ and $\Delta / \lambda_{T S}$ are fixed, $A_{0} /(a h) \propto M /(1+M) \lambda(M)$, which is in good qualitative agreement with the Navier-Stokes results both for $R e=8.4 \times 10^{6}$ and $R e=35.1 \times 10^{6}$. Note that the factor $M /(1+M)$ comes from equation (3.9b); therefore, since in our case $\lambda$ decreases with increasing Mach number, the enhanced receptivity observed in figure 7 (c) for increasing $M$ is due to the fact that the amplitude of the near wall $u$-velocity disturbances induced by the acoustic wave increases with Mach number.

It is important to note that, in all the results presented here, there is a substantial discrepancy between the theoretically predicted lower branch T-S wavelength $\lambda_{T S}=$ $R e^{-3 / 8} \mu_{w}^{-1 / 4} \rho_{w}^{-1 / 2} \lambda^{-5 / 4} \beta^{-3 / 4} 2 \pi / \bar{\alpha}_{1}$ and that obtained from LST. As an example, consider the cases shown in figure 7 (a). In this case the asymptotic theory gives $\lambda_{T S}=0.0378$ while from LST $\lambda_{T S}=0.0285$, and hence there is a relative error of about $24.6 \%$, which increases with decreasing Reynolds number. Plotting the data in figure 7 (a) against $\Delta$, instead of $\Delta / \lambda_{T S}$, would result in a disagreement, between the theory and the numerical results, regarding the position (in $\Delta$ ) of the maximum T-S wave amplitude. This discrepancy, and the associated error in T-S wave amplitude, are eliminated by interpreting the theoretical results in terms of the parameter $\Delta / \lambda_{T S}$. One can then obtain $\lambda_{T S}$ using LST and recover the $\Delta / \lambda_{T S}$ needed to calculate the Fourier transform of the roughness shape, which in our case is given by equation (5.5).

\subsection{Lower branch $T$-S wave receptivity due to a vibrating ribbon}

The numerical analysis of the vibrating ribbon receptivity problem is carried out by subjecting the laminar boundary layer flow to wall disturbances given by (4.9). Again, in order to be consistent with the nomenclature used for the derivation of the theoretical results, the flow is decomposed as

$$
u=U_{B}+u_{1},
$$

where, as usual, $U_{B}$ is the laminar boundary layer flow and $u_{1}$ denotes the disturbance field. An example of the disturbances introduced in the flow due to the vibrations of the ribbon placed at the wall is given in figure 8 through instantaneous contours of $p_{1}$, showing that the vibrating ribbon leads to the excitation of a $\mathrm{T}-\mathrm{S}$ wave propagating in the flow direction. The T-S wave amplitude function, extracted from the Navier-Stokes results at $x=340$, is compared in figure 9 (a) with the corresponding T-S wave eigenfunction obtained by LST, showing a virtually perfect match. As for the acoustic wave receptivity study, the amplitude of the wall-pressure disturbances is calculated by making use of the discrete Fourier transform formula (5.2) and the initial T-S wave amplitude $A_{0}$ is obtained by combining the Navier-Stokes result with the T-S wave growth predicted by LST. Figure 9(b) shows the results obtained for cases M0.2A1, M0.2B and M0.2E. The 


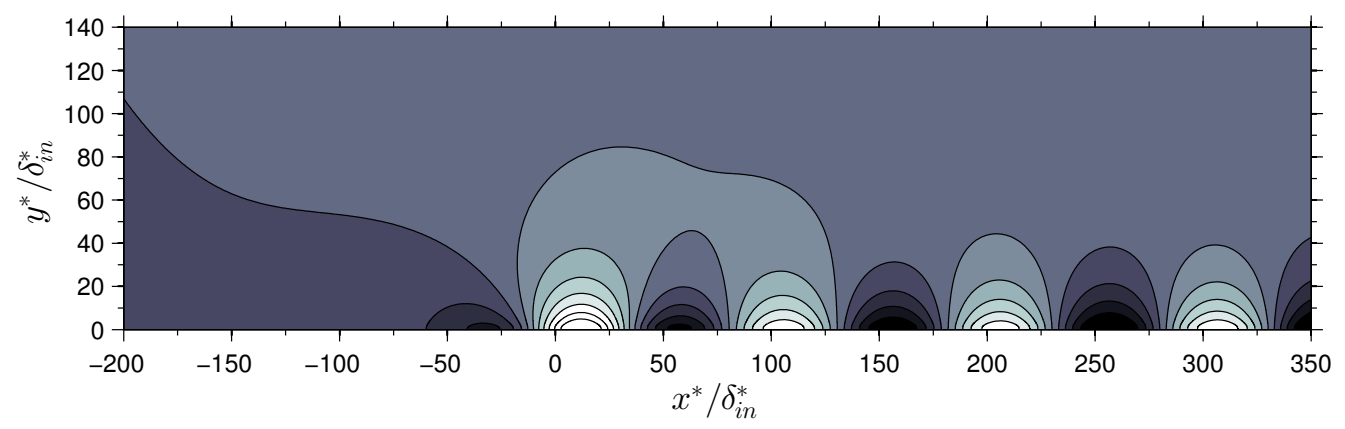

FiguRE 8. Boundary layer response due to the vibrations of a ribbon positioned at the flat plate wall for case M0.2A1. The plot show contours of instantaneous pressure disturbance $p_{1}$.

(a)

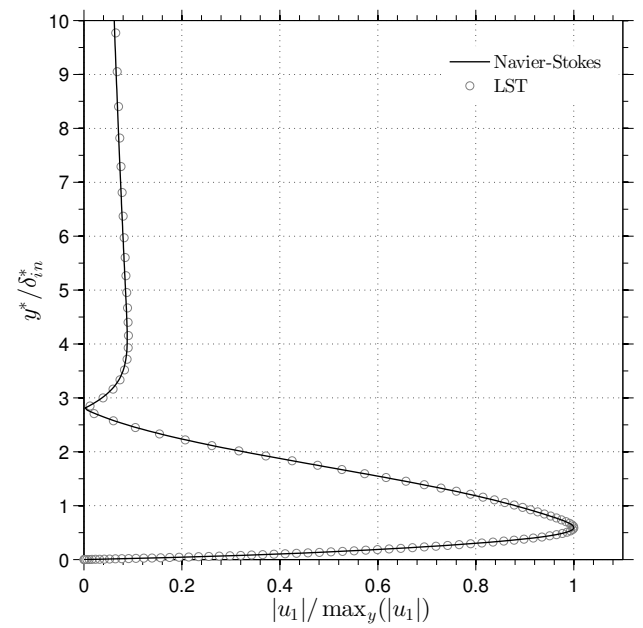

(b)

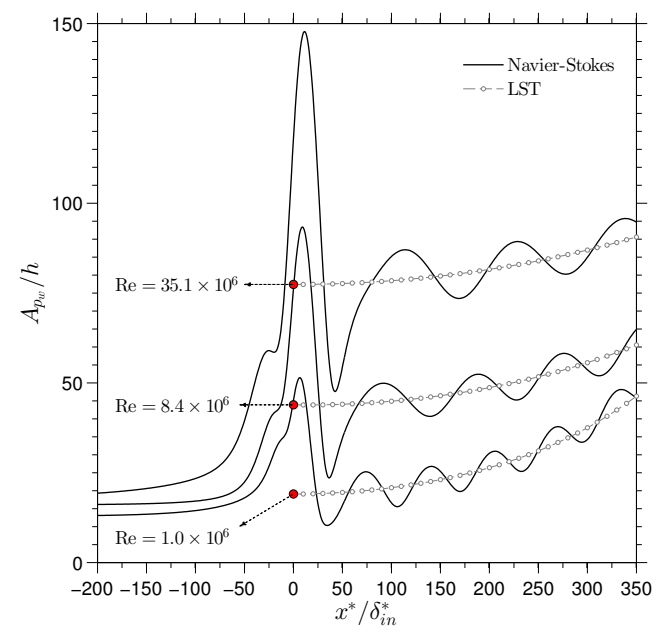

Figure 9. Comparing the linearised Navier-Stokes results with LST. (a) The $u$-velocity amplitude function at $x=340$ of the T-S wave generated by the vibrating ribbon for case M0.2A1. (b) Streamwise variation of the amplitude of the wall-pressure perturbation induced by the vibrating ribbon for cases $\mathrm{M} 0.2 \mathrm{~A} 1, \mathrm{M} 0.2 \mathrm{~B}$ and $\mathrm{M} 0.2 \mathrm{E}$.

first thing to note is that, in addition to the T-S wave, the vibrating ribbon also excites an acoustic wave, as can be inferred from the non-zero wall-pressure amplitude upstream of the vibrating ribbon. In addition, the superposition of the acoustic wave with the T-S wave leads to a periodic beating of the wall-pressure amplitude downstream of the vibrating ribbon. As a consequence, in order to extract the T-S wave amplitude $A_{0}$ from the Navier-Stokes results, the amplitude growth predicted by LST is scaled to match, at $x^{*} / \delta_{i n}^{*}=200$, the curve obtained by averaging between the maximum and minimum amplitude envelopes. The amplitudes extracted using this procedure are denoted by greyfilled circles (red online) in figure $9(\mathrm{~b})$. Now, recall that the asymptotic theory gives

$$
\begin{aligned}
& p_{1 w}=\epsilon R e^{-1 / 4} \frac{\mu_{w}^{1 / 2}}{\lambda^{-1 / 2} \beta^{1 / 2}}\left\{\frac{1}{2} \check{p}_{1 w} \mathrm{e}^{\mathrm{i} \omega t}+\text { c.c. }\right\} \\
& \check{p}_{1 w}=-\sqrt{2 \pi} \mathcal{C}_{v} \hat{F}\left(\bar{\alpha}_{1}\right) \mathrm{e}^{\mathrm{i} \bar{\alpha}_{1} \bar{x}}
\end{aligned}
$$




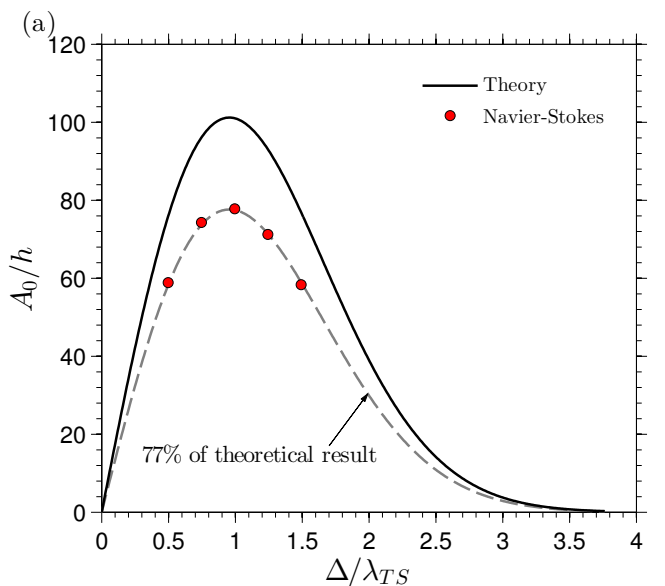

(b)

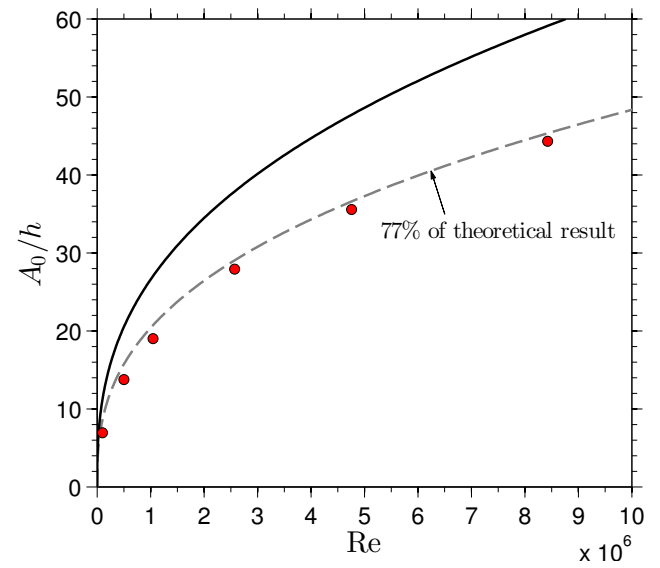

(c)

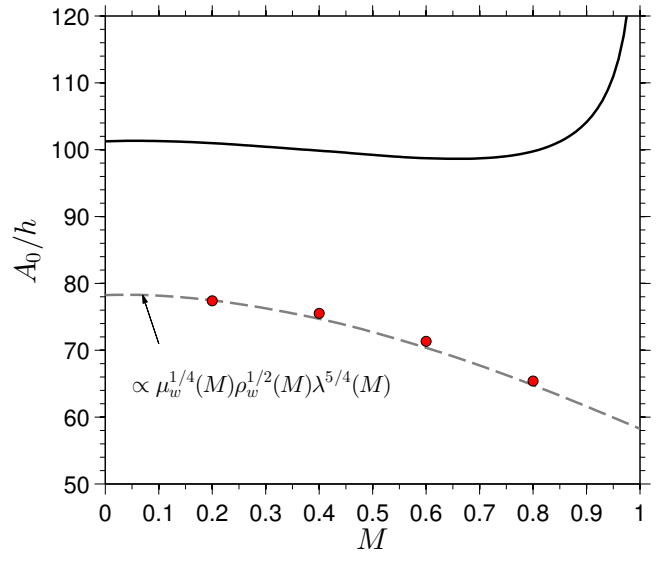

Figure 10. Comparisons between the asymptotic theory and the Navier-Stokes simulations for the acoustic wave receptivity study, showing the variation of initial T-S wave amplitude as a function of: (a) $\Delta / \lambda_{T S}$ for $R e=35.1 \times 10^{6}$ and $M=0.2$ (numerical cases M0.2A1, M0.2A2, M0.2A3, M0.2A4 and M0.2A5); (b) Re for $M=0.2$ and $\Delta / \lambda_{T S}=1$ (numerical cases M0.2B, $\mathrm{M} 0.2 \mathrm{C}, \mathrm{M} 0.2 \mathrm{D}, \mathrm{M} 0.2 \mathrm{E}, \mathrm{M} 0.2 \mathrm{~F}$ and $\mathrm{M} 0.2 \mathrm{G}$ ); (c) $M$ for $\Delta / \lambda_{T S}=1$ and $R e=35.1 \times 10^{6}$ (numerical cases M0.2A1, M0.4A, M0.6A and M0.8A)

where $\mathcal{C}_{v}$ and $\hat{F}\left(\bar{\alpha}_{1}\right)$ are given by (3.18) and (5.5), respectively. Therefore we may write

$$
\frac{A_{0}}{h}=\underbrace{R e^{5 / 8}}_{\epsilon / h} R e^{-1 / 4} \frac{\mu_{w}^{1 / 2}}{\lambda^{-1 / 2} \beta^{1 / 2}}\left|\check{p}_{1 w}\right| .
$$

Comparisons between the theoretical and numerical results are shown in figure 10. Figure 10(a) gives the T-S wave amplitude variation as a function of $\Delta / \lambda_{T S}$ for fixed Reynolds number, Mach number and wall-temperature. It can be seen that, again, the T-S wave receptivity is only a function of the roughness shape Fourier coefficient corresponding to the wavenumber of the T-S wave and the maximum receptivity is obtained for $\Delta / \lambda_{T S}=3 / \pi$. The relative error between the theory and the Navier-Stokes simulations is about $23 \%$ of the theoretical result in all cases. Figure 10(b) shows the T-S wave amplitude variation as a function of Reynolds number. The asymptotic theory indicates that the initial T-S wave amplitude is proportional to $R e^{3 / 8}$ (see 5.9). The agreement be- 
(a)

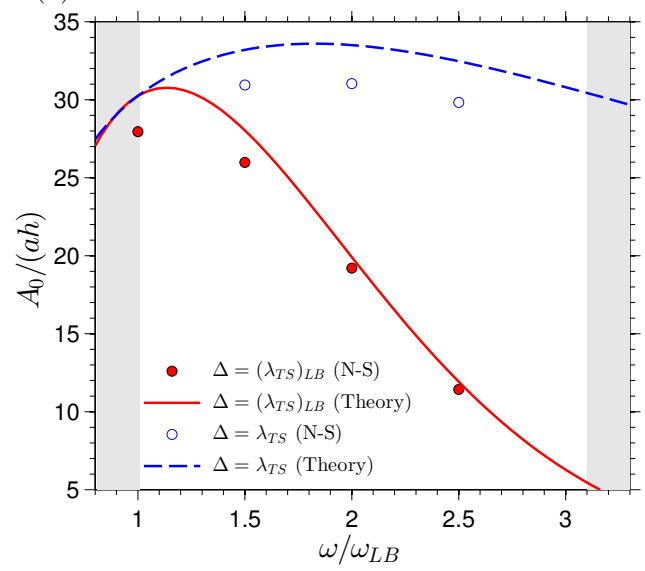

(b)

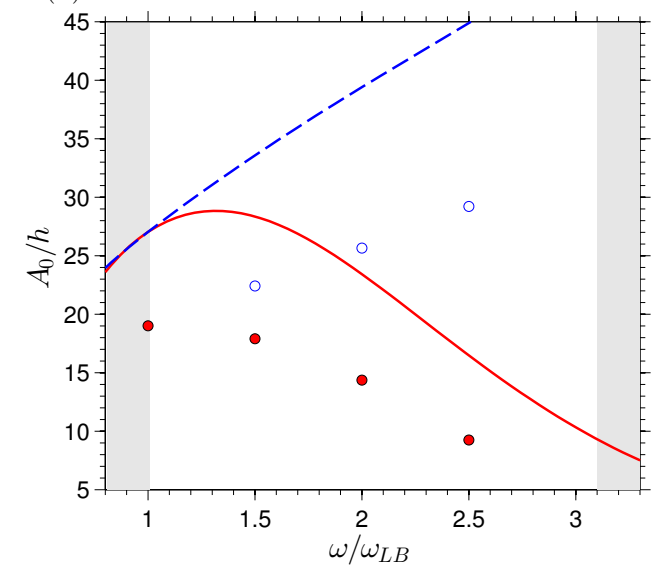

Figure 11. Comparisons between the asymptotic theory and the Navier-Stokes simulations, showing the variation of initial T-S wave amplitude as a function of frequency. The grey areas delimit the range of unstable frequencies as predicted by LST. (a) Acoustic wave receptivity, (b) vibrating ribbon receptivity.

tween theory and numerical simulations is good, with the $23 \%$ relative error being nearly constant over the whole Reynolds number range considered. The T-S wave amplitude variation as a function of Mach number is shown in figure 10(c). It is important to note that, for fixed $R e$ and $\Delta / \lambda_{T S}$, the theory shows that

$$
\frac{A_{0}}{h} \propto \frac{\mu_{w}^{1 / 4}(M) \rho_{w}^{1 / 2}(M)}{\lambda^{-5 / 4}(M) \beta^{1 / 4}(M)},
$$

where the Mach number dependence for $\rho_{w}$ and $\mu_{w}$ appears because we take the walltemperature to be specified according to (2.5). Therefore, the theoretical result presents a singularity at $M=1$ (i.e. $\beta=0$ ), and hence numerical simulations and theory quickly depart from each other as $M \rightarrow 1$. Interestingly, however, the results also show that the different T-S wave amplitudes obtained numerically for different Mach numbers follow closely the behaviour predicted by (5.10) after neglecting the contribution from $\beta$ to the wall-pressure disturbance associated with the T-S wave, as shown by the dashed line in figure $10(\mathrm{c})$.

\subsection{Effects of disturbance frequency and roughness height}

In the previous two sections we have shown that the predictions of the asymptotic theories developed by Ruban (1984), Goldstein (1985) and Terent'ev (1981) compare well with Navier-Stokes results for cases regarding the linear receptivity of lower branch T-S waves. The analysis was restricted to these cases because, strictly speaking, triple-deck theory only describes T-S waves near the lower branch of the neutral stability curve (Smith $1979 a, b)$. In this section, the analysis will be extended by looking at how the theoretical predictions compare with Navier-Stokes results as the frequency is increased within the unstable T-S wave range. In addition, we will estimate the critical roughness height for which the T-S wave receptivity by sound/roughness interaction first becomes nonlinear, and hence can no longer be predicted by a linear theory.

Figures 11(a) and 11(b) show a comparison of the T-S wave amplitude variation as a function of frequency, at $M=0.2$ and $R e=1 \times 10^{6}$, for the acoustic and vibrating ribbon receptivity cases, respectively. The figures report two sets of results: the dashed blue lines 


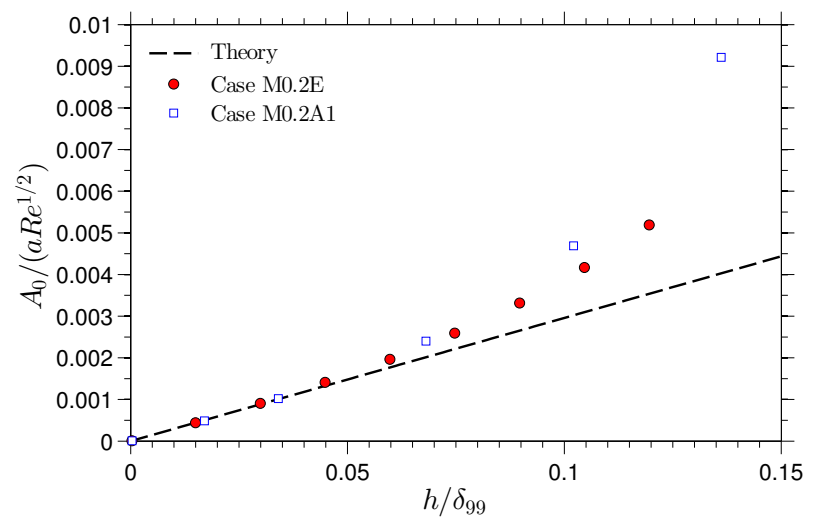

FiguRE 12. Comparisons between the asymptotic theory and the Navier-Stokes simulations for the acoustic wave receptivity study, showing the variation of initial T-S wave amplitude as a function of roughness height.

and the blue circles are for wall disturbances with width $\Delta$ equal to the wavelength of the excited T-S wave; the continuous red lines and the red dots are for wall disturbances with $\Delta$ equal to the lower branch T-S wavelength. It can be seen that, in all cases, the variation of T-S amplitude as a function of frequency is captured accurately by the asymptotic theory. Interestingly, the agreement between the theoretical predictions and the NavierStokes results remains good for frequencies well above the lower branch frequency. This is especially true for the acoustic wave/roughness cases, while the agreement deteriorates slightly as the frequency increases for the vibrating ribbon receptivity cases. Note that, according to LST, the upper branch of the neutral curve is located at $\omega / \omega_{L B} \approx 3.1$, where $\omega_{L B}$ is the lower branch frequency, for the flow parameters considered. The calculation of the unstable T-S wave amplitudes predicted by the asymptotic theories is carried out by solving equation (3.13) for a chosen real frequency $\bar{\omega}$ to obtain the associated complex $\bar{\alpha}$ and $z$, which are then used in equations (3.11) and (3.17) to obtain the T-S wave amplitudes. Note that the results in figure 11 are plotted against $\omega / \omega_{L B}$ in order to minimise errors originating from the calculation of the lower branch frequency. In fact, similarly to what was found for $\lambda_{T S}$ in $\S 5.1$, there is a substantial discrepancy between the theoretically predicted $\omega_{L B}=R e^{1 / 4} \mu_{w}^{1 / 2} \lambda^{3 / 2} \beta^{1 / 2} \bar{\omega}_{1}$ and that obtained from LST.

Finally, the variation of T-S wave amplitude as a function of roughness height $h$ for cases M0.2E and M0.2A1 is compared with the linear asymptotic theory result in figure 12. The plot shows numerical results for two different Reynolds numbers, namely $R e=1.0 \times 10^{6}$ (case M0.2E) and $R e=35.1 \times 10^{6}$ (case M0.2A1). Note that, having normalised $A_{0}$ using $R e^{1 / 2}$, the theoretical result becomes independent of Reynolds number. The figure clearly shows that for $h / \delta_{99}<0.06$, where $\delta_{99}$ is the boundary layer thickness at the centre of the roughness element, the numerical results follow closely the linear behaviour predicted by the asymptotic theory. On the other hand, the discrepancy between theoretical and numerical results increases rather quickly starting from $h / \delta_{99} \approx 0.06$, indicating that nonlinear effects start becoming important for $h \approx 0.06 \delta_{99}\left(\right.$ or $\left.h \approx 0.17 \delta^{*}\right)$. This result is in very good agreement with that reported by Choudhari \& Street (1992) and Crouch (1992) when comparing their finite-Reynolds number Orr-Sommerfeld receptivity theory results with the experiments of Saric et al. (1991), which were carried out for a Reynolds number of $R e=3.39 \times 10^{5}$. Interestingly, these results suggest that the critical value of $h / \delta_{99}$, needed for nonlinear receptivity, is little affected by the Reynolds number. 


\section{Conclusions}

The receptivity of Tollmien-Schlichting waves in subsonic boundary layers has been investigated using triple-deck theory and numerical simulations of the compressible NavierStokes equations. The investigation focused on the two receptivity problems arising due to the interaction between an acoustic wave and a small isolated roughness element placed on the wall of a flat plate and due to the time-periodic vibrations of a ribbon placed on the wall of an otherwise smooth flat plate. A parametric study looking at the effects of roughness element and vibrating ribbon longitudinal dimensions, Reynolds number, and Mach number was performed in order to thoroughly investigate the accuracy of the asymptotic theory for the prediction of lower branch T-S wave receptivity in subsonic boundary layers.

The results show that the theoretical predictions are in good agreement with the Navier-Stokes results. For both the receptivity problems analysed, the amplitude $A_{0}$ of the excited T-S wave was found to be dependent on the Fourier transform of the wall disturbance (i.e. the roughness element or the vibrating ribbon) rather than being directly affected by its shape. In particular, only the Fourier coefficient corresponding to the T-S wave wavenumber is responsible for the receptivity process. In the case of the $\mathrm{T}-\mathrm{S}$ wave receptivity due to sound, both the theoretical and numerical results indicate that the T-S wave amplitude increases with Reynolds number proportionally to $R e^{1 / 2}$. In this case the agreement between theory and Navier-Stokes simulations is excellent. Despite the theoretical results being derived for large values of the Reynolds number, the numerical simulations show that the theory performs well for Reynolds numbers down to at least $R e=1.0 \times 10^{5}$; the relative error between theory and numerical simulations was found to be about $7 \%$ of the theoretical result for Reynolds numbers between $1.0 \times 10^{6}$ and $35.1 \times 10^{6}$ at $M=0.2$. As the Mach number increases, the T-S wave receptivity is enhanced mainly due to the higher near wall $u$-velocity disturbances induced by the acooustic wave at higher free stream Mach numbers. The asymptotic theory suggests that the T-S wave amplitude increases according to $A_{0} /(a h) \propto M /(1+M) \lambda(M)$, which agrees qualitatively with the numerical results obtained for both $R e=8.4 \times 10^{6}$ and $R e=35.1 \times 10^{6}$. However, the relative error increases slightly for increasing Mach number, reaching a maximum of about $13.5 \%$ at $M=0.8$.

The results obtained for the vibrating ribbon receptivity problem indicate that the amplitude of the excited T-S wave is proportional to $R e^{3 / 8}$. In this case the relative error between theory and Navier-Stokes simulations is about $23 \%$ of the theoretical result and remains nearly constant for Reynolds numbers between $1.0 \times 10^{6}$ and $35.1 \times 10^{6}$ at $M=0.2$. The agreement between theory and Navier-Stokes simulations remains good also in this case, especially considering that the theoretical results were derived by keeping only the leading order terms in the asymptotic expansions. When looking at how the receptivity varies with Mach number it was found that, while the numerical simulations show that the T-S wave amplitude decreases as the Mach number increases, the theoretically predicted wall-pressure presents a singularity for $M=1$ and, as a consequence, numerical and theoretical results quickly depart from each other as $M \rightarrow 1$. The singularity appears in the form of the factor $\beta^{-1 / 4}$ and originates in the upper-deck. Despite this singularity, it was found that the amplitude variation with Mach number extracted from the numerical results follows closely that obtained from the theoretical result after neglecting the contribution from $\beta$ to the pressure disturbance at the wall, so that the T-S wave amplitude varies as $A_{0} / h \propto \mu_{w}^{1 / 4}(M) \rho_{w}^{1 / 2}(M) \lambda^{5 / 4}(M)$.

Additional numerical simulations indicate that the asymptotic theory can also be used to predict the receptivity of unstable T-S waves with good accuracy. In addition, in 
agreement with the findings of Choudhari \& Street (1992) and Crouch (1992), we show that the receptivity process due to the acoustic wave/roughness interaction is linear provided the height of the roughness element is smaller than about $6 \%$ of the local boundary layer thickness.

The theoretical triple-deck results are obtained under several assumptions regarding the order of magnitude of the size, amplitude and frequency of the external disturbances. These assumptions are needed in order to arrive at the asymptotic solutions for large values of the Reynolds number. However, the numerical results presented in this work show that the behaviour of the solutions obtained for $R e \rightarrow \infty$ holds also for finite Reynolds numbers and, in practise, the only conditions to be met in order for the theory to give reliable predictions are that the amplitude of the disturbances considered (acoustic wave, roughness element and vibrating ribbon for the cases analysed here) be small enough for the receptivity process to be linear and that the free stream Mach number be subsonic. As such, it appears clear that the asymptotic theory of receptivity represents a good candidate for providing the amplitude information missing in current laminarturbulent transition prediction methods.

\section{Acknowledgments}

This research was performed in the Laminar Flow Control Centre (LFC-UK) at Imperial College London. The Centre is supported by EPSRC, Airbus UK and EADS Innovation Works. The numerical simulations were performed in the Imperial College cx1 cluster. The authors would like to thank Prof Neil D. Sandham for providing the SBLI code used for the numerical study and the anonymous referees for their constructive comments.

\section{REFERENCES}

Balakumar, P. \& Malik, M. R. 1992 Discrete modes and continuous spectra in supersonic boundary layers. J. Fluid Mech. 239, 631-656.

Borodulin, V. I., Ivanov, A. V., Kachanov, Y. S. \& Roschektaev, A. P. 2013 Receptivity coefficients at excitation of cross-clow waves by free-stream vortices in the presence of surface roughness. J. Fluid Mech. 716, 487-527.

Carpenter, M. H., Nordstrom, J. \& Gottlieb, D. 1999 A stable and conservative interface treatment of arbitrary spatial accuracy. Journal of Computational Physics 148, 341-365.

Choudhari, M. \& Street, C. L. 1992 A finite Reynolds-number approach for the prediction of boundary-layer receptivity in localized regions. Physics of Fluids A 4 (11), 2495-2514.

Crouch, J. D. 1992 Localized receptivity of boundary layers. Physics of Fluids A 4 (7), 14081414.

De Tullio, N. 2013 Receptivity and transition to turbulence of supersonic boundary layers with surface roughness. PhD thesis, School of Engineering Sciences, University of Southampton.

De Tullio, N., Paredes, P., Sandham, N. D. \& Theofilis, V. 2013 Laminar-turbulent transition induced by a discrete roughness element in a supersonic boundary layer. J. Fluid Mech. 735, 613-646.

De Tullio, N. \& Sandham, N. D. 2010 Direct numerical simulation of breakdown to turbulence in a Mach 6 boundary layer over a porous surface. Physics of Fluids 22 (094105).

Denier, J. P., Hall, P. \& Seddougui, S. O. 1991 On the receptivity problem for Görtler vortices: vortex motion induced by wall roughness. Phil. Trans. R. Soc. A 335, 51-85.

Dietz, A. J. 1999 Local boundary-layer receptivity to a convected free-stream disturbance. $J$. Fluid Mech. 378, 291-317.

Duck, P. W., Ruban, A. I. \& Zhikharev, C. N. 1996 The generation of Tollmien-Schlichting waves by free stream turbulence. J. Fluid Mech. 312, 341-371. 
Fucciarelli, D., Reed, H. \& Lytthe, I. 2000 Direct numerical simulation of leading-edge receptivity to sound. AIAA J. 38 (7), 1159-1165.

Goldstein, M. \& Hultgren, L. S. 1987 A note on the generation of Tollmien-Schlichting waves by sudden surface-curvature change. J. Fluid Mech. 181, 519-525.

Goldstein, M. E. 1985 Scattering of acoustic waves into Tollmien-Schlichting waves by small streamwise variations in surface geometry. J. Fluid Mech. 154, 509-529.

Jones, R. D., Sandberg, R. D. \& SAndham, N. D. 2010 Stability and receptivity characteristics of a laminar separation bubble on an aerofoil. J. Fluid Mech. 648, 257-296.

Kachanov, K., Kozlov, V. V. \& Levchenko, V. Y. 1979 Origin of Tollmien-Schlichting waves in boundary layer under the influence of external disturbances. Fluid Dyn. 13, 704711.

Kachanov, Y. S. 1994 Physical mechanisms of laminar-boundary-layer transition. Ann. Rev. Fluid Mech. 26, 411-482.

Kerimbekov, R. M. \& Ruban, A. I. 2005 Receptivity of boundary layers to distributed wall vibrations. Phil. Trans. R. Soc. A 363, 1145-1155.

Kozlov, V. V. \& Ryzhov, O. S. 1990 Receptivity of boundary layers: asymptotic theory and experiment. Phil. Trans. R. Soc. A 429, 341-373.

Lin, C. C. 1946 On the stability of two-dimensional parallel flows. Part 3. Stability in a viscous fluid. Q. Appl. Maths 3, 277-301.

Reshotкo, E. 1976 Boundary layer stability and transition. Ann. Rev. Fluid Mech. 8, 311-349.

REYNOLDS, O. 1883 An experimental investigation of the circumstances which determine whether the motion of water shall be direct or sinuous, and of the law of resistance in parallel channels. Phil. Trans. R. Soc. 174, 935-82.

RubAn, A. I. 1984 On the generation of Tollmien-Schlichting waves by sound. Izv. Akad. Nauk SSSR Mekh. Zhidk. Gaza 5, 44-52 (English translation Fluid Dyn. 19(5) 709-717, 1984).

Ruban, A. I., Bernots, T. \& Pryce, D. 2013 Receptivity of the boundary layer to vibrations of the wing surface. J. Fluid Mech. 723, 480-528.

Sandham, N. D., Li, Q. \& Yee, H. C. 2002 Entropy splitting for high-order numerical simulation of compressible turbulence. Journal of Computational Physics 178, 307-322.

Saric, W. S., Hoos, J. A. \& Radeztsky, R. H. 1991 Boundary-layer receptivity of sound with roughness. In Boundary Layer Stability and Transition to Turbulence, pp. 17-22. ASME, New York.

Saric, W. S., Reed, H. L. \& Kerschen, E. J. 2002 Boundary-layer receptivity to freestream disturbances. Ann. Rev. Fluid Mech. 34, 291-319.

SARIC, W. S. \& White, E.B. 1998 Influence of high- amplitude noise on boundary-layer transition to turbulence.

Schubauer, G. B. \& Skramstad, H. K. 1948 Laminar boundary layer oscillations and transition on a flat plate. Tech. Rep. 909. NACA.

Smith, F. T. $1979 a$ Nonlinear stability of boundary layers for disturbances of various sizes. Proc. R. Soc. Lond. A 368, 573-589.

Smith, F. T. $1979 b$ On the nonparallel flow stability of the Blasius boundary layer. Proc. $R$. Soc. Lond. A 366, 91-109.

Tempelmann, D., Schrader, L. U., Hanifi, A., Brandt, L. \& Henningson, D. S. 2012 Swept wing boundary-layer receptivity to localized surface roughness. J. Fluid Mech. 711, 516-544.

Terent'Ev, E. D. 1981 Linear problem of a vibrator in subsonic boundary layer. Prikl. Mat. Mekh. 45, 1049-1055 (English translation Appl. Math. Mech. 45(6), 791-795, 1981).

Thomson, K. W. 1987 Time dependent boundary conditions for hyperbolic systems. Journal of Computational Physics 68, 1-24.

Thomson, K. W. 1990 Time dependent boundary conditions for hyperbolic systems, II. Journal of Computational Physics 89, 439-461.

Wanderley, J. B. \& Corke, T. C. 2001 Boundary layer receptivity to free-stream sound on elliptic leading edges of flat plates. J. Fluid Mech. 429, 1-21.

White, F. M 2005 Viscous fluid flow, $3^{\text {nd }}$ edn. McGraw-Hill.

WrAY, A. A. 1990 Minimal storage time advancement schemes for spectral methods. Rept. M.S. 202 A-1. NASA Ames Research Centre.

Wu, X. 2001 Receptivity of boundary layers with distributed roughness to vortical and acoustic 
disturbances; a second-order theory and comparisons with experiments. J. Fluid Mech. 431, 91-133.

Wu, X., Zhao, D. \& Luo, J. 2011 Excitation of steady and unsteady Görtler vortices by free stream vortical disturbances. J. Fluid Mech. 682, 66-100.

Zhigulev, V. N. \& Fedorov, A. V. 1987 Boundary-layer receptivity to acoustic disturbances. Journal of Applied Mechanics and Technical Physics 28 (1), 28-34. 